This document is the accepted manuscript version of the following article:

Gyse1, M., McFiggans, G. B., \& Coe, H. (2009). Inversion of tandem

differential mobility analyser (TDMA) measurements. Journal of Aerosol

Science, $40(2), 134-151$.

https://doi .org/10.1016/j.jaerosci .2008.07.013

This manuscript version is made available under the CC-BY-NC-ND 4.0

license http://creativecommons.org/1icenses/by-nc-nd/4.0/

\title{
Inversion of Tandem Differential Mobility Analyser (TDMA) Measurements
}

\author{
M. Gysel ${ }^{\mathrm{a}, \mathrm{b}, *, 1}$ G. B. McFiggans ${ }^{\mathrm{b}}$ H. Coe ${ }^{\mathrm{b}}$ \\ ${ }^{a}$ Laboratory of Atmospheric Chemistry, Paul Scherrer Institut, 5232 Villigen PSI, \\ Switzerland \\ ${ }^{\mathrm{b}}$ School of Earth, Atmospheric $\& 3$ Environmental Sciences, The University of \\ Manchester, Simon Building, Oxford Road, Manchester, M13 9PL, UK
}

\begin{abstract}
The tandem differential mobility analyser (HTDMA) technique is used to detect size changes of submicron particles after a treatment such as exposure to high relative humidity. Measured diameter growth factor distributions must be inverted, because they are only a skewed and smoothed integral transform of the actual growth factor probability density function (GF-PDF). We introduce a new approach, TDMAinv, representing the inverted GF-PDF as a piecewise linear function. Simulated measurements are used to prove the concept. Measurements of an aerosol with a bimodal GF-PDF show that TDMAinv provides equivalent information to TDMAfit, the most widely used inversion algorithm. The major advantage of TDMAinv is that convergence of the inversion is robust and independent of the initial guess. This makes TDMAinv a reliable tool to analyse large TDMA data sets. A methodology is also demonstrated for analysis of TDMA data in cases where the dominant fraction of selected particles is doubly or triply charged.
\end{abstract}

Key words: DMA, HTDMA, Inversion, Hygroscopicity, TDMAinv, TDMAfit

\section{Introduction}

Diameter changes of submicron particles can be precisely determined using the Tandem Differential Mobility Analyser (TDMA) method introduced by

\footnotetext{
* corresponding author

Email address: martin.gysel@psi.ch (M. Gysel).

URL: http://people.web.psi.ch/gysel/ (M. Gysel).

1 Phone: +41563104168
} 
Liu et al. (1978). The most widespread application is the hygroscopicity or humidified (H-)TDMA, where the dry selected particles are exposed to a well defined relative humidity $(\mathrm{RH})$, in order to measure hygroscopic growth factor distributions (e.g. Swietlicki et al., 2008; Gysel et al., 2002; Rader and McMurry, 1986; Liu et al., 1978). The reaction rate of gaseous ammonia with sulphuric acid aerosols has been investigated by McMurry et al. (1983) using a flow tube reactor in between the two differential mobility analysers (DMA). Joutsensaari et al. (2001) have developed an organic (O-)TDMA, in order to determine the affinity of particles to different organics by exposing them to e.g. subsaturated ethanol vapours. Evaporation rates of volatile particles at ambient temperature have been determined by installation of a laminar flow cell in between the two DMAs (e.g. Bilde and Pandis, 2001; Dassios and Pandis, 1999). The volatility (V-)TDMA technique is used to measure the amount of refractory material in aerosol particles (e.g. Paulsen et al., 2006; Philippin et al., 2004), while the combined volatilisation humidification (VH-)TDMA technique is used to investigate the contributions of different constituents in mixed particles to the hygroscopic water uptake (e.g. Johnson et al., 2004).

The measurement signal of a TDMA, hereinafter referred to as the measurement distribution function (MDF), is the particle concentration at the TDMA outlet as a function of growth factor (GF) set at the instrument. The MDF is only a skewed and smoothed integral transform of the particle's actual growth factor probability density function (GF-PDF). Therefore an inversion algorithm has to be applied to the MDF of TDMA measurements to retrieve the GF-PDF. Key goals of TDMA data analysis approaches are to determine the mean GF of the sample, to provide the number fractions of particles in different GF ranges and to retrieve the correct shape of the GF-PDF in detail. Different existing approaches can be grouped into three distinct methods (Table 1). The simplest method is to use the MDF without any data inversion to determine integral properties of the GF-PDF(e.g. Liu et al., 1978; Weingartner et al., 2002). Generally, this approach does not fulfil any specification listed in Table 1. An exception are samples exhibiting monomodal growth with limited spread between individual particles, where the MDF can be used to determine the mean GF. A second approach is to invert for the smearing and skewing effect of the second DMA only, in order to recover the particle size distribution after treatment (e.g. Cocker III et al., 2001; Stratmann et al., 1997; Voutilainen et al., 2000). This approach fulfils all specifications because the particle size distribution after treatment resembles the GF-PDF except for some smoothing caused by the finite width of the size cut of particles selected with DMA1. The third class of methods aims to recover the actual GF-PDF by inversion of the MDF using a complete TDMA forward function (e.g. Cubison et al., 2005; Stolzenburg and McMurry, 1988). This class of methods satisfies all criteria as closely as possible within the limits imposed by the measurement uncertainties and inversion algorithm itself. 
The TDMA measurement technique is widely used nowadays. However, the raw measurement data are often not properly inverted. Reliable and automated inversion algorithms which are freely available are therefore of great need, particularly for long-term ambient measurements, where the mixing state of the aerosol is unknown. The TDMAfit algorithm (e.g. Stolzenburg and McMurry, 1988) is the most widespread inversion approach. It describes the GF-PDF as a superposition of multiple Gaussians, whereas the mean GF, standard deviation and number fraction in each Gaussian mode are varied until the observed MDF is reproduced by sending the resulting GF-PDF through the forward function of the TDMA. However, convergence of fitting multiple modes is not robust in cases with largely overlapping modes or shoulders, and successful convergence may depend on the initial guess. This makes automated data analysis of large data sets difficult. The optimal estimation method (OEM), recently introduced by Cubison et al. (2005), uses a quasi-inverse matrix of the TDMA kernel function in order to retrieve the values of the GF-PDF at discrete bin positions. This method is very efficient, returns unambiguous results for given retrieval bins and provides tools for a thorough uncertainty analysis. However, constraints such as keeping the GF-PDF positive can not be applied because it is a linear method. This can lead to oscillations with alternating positive and negative values in the retrieved GF-PDF, which propagate outside of the support of the MDF, if the chosen resolution is too high (see Sect. 3.1).

The requirements and issues depicted above were the motivation to develop an alternative TDMA data inversion approach, hereinafter referred to as TDMAinv. TDMAinv is a robust and automated data inversion algorithm, which has successfully been tested and applied to large HTDMA data sets in recent laboratory and field studies (Meyer et al., 2008; Sjogren et al., 2008; Allan et al., 2008; Gysel et al., 2007).

\section{Inversion approach}

\subsection{TDMA measurement technique}

A Differential Mobility Analyser (DMA; Knutson and Whitby, 1975; Liu and Pui, 1974) allows selecting a narrow size cut from a polydisperse aerosol in the sub- $\mu$ m diameter range. Two DMAs operated in series with a treatment unit in between form the core of a TDMA instrument (Fig. 1). The treatment unit can consist of e.g. a humidifier, thermodesorber, flow tube, chemical reactor, organic vapour conditioner or a combination of such elements. The investigated aerosol is typically first dried and brought to charge equilibrium before a monodisperse size cut of well known mobility diameter is selected using the 
first DMA. These particles then undergo a treatment, before a second DMA combined with a condensation particle counter (CPC) is used to determine the resulting particle size. The ratio of the diameter after treatment to the initially selected diameter is defined as the growth factor, GF. Note, particles may grow or shrink depending on the applied treatment and therefore measured GFs can be $>1$ or $<1$, respectively. An MDF covering the whole GF range is obtained by changing the diameter selected with DMA2 gradually or in discrete steps, while keeping DMA1 at a constant dry size. The MDF is only a smoothed and skewed integral transform of the particle's actual GF-PDF because the transfer functions of the DMAs are of finite width and because the TDMA's overall transfer probability depends on the GF of the particles (see Appendix B.1 and Fig. 2). Furthermore the MDF may contain contributions from particles with larger dry diameter carrying multiple charges, which have an electrical mobility matching the diameter set at DMA1 for singly charged particles. Appropriate inversion of the raw measurement data is therefore required in most TDMA applications, in order to recover the actual GF-PDF of the investigated aerosol.

\subsection{TDMAinv algorithm}

The physics of a TDMA instrument is described by the two-dimensional TDMA kernel function, $K_{T D M A}\left(g_{s e t}, \hat{g}\right)$, which is the instrument response, $m_{T D M A}$, as a function of GF set at the TDMA, $g_{\text {set }}$, if all particles of the selected dry size exhibit a true $\mathrm{GF}=\hat{g}$, that is if the GF-PDF, $c(g)$, is a Dirac's delta function with peak at $\mathrm{GF}=\hat{g}$ :

$$
K_{T D M A}\left(g_{\text {set }}, \hat{g}\right)=\frac{m_{T D M A}\left(g_{\text {set }}\right)}{N_{\text {inlet }}} \quad \text { for } c(g)=\delta(g-\hat{g})
$$

Detailed equations for the kernel function and the normalisation factor, $N_{\text {inlet }}$, are provided in Appendix B.1. $K_{T D M A}$ is the kernel of the integral transform, which allows calculation of the MDF measured by a TDMA for any given GF-PDF. The TDMA response is proportional to the number concentration of charged particles in the relevant electrical mobility size range, and it is linear in $c$, i.e. $\left.m_{T D M A}\left[a c_{1}+(1-a) c_{2}\right)\right]=a m_{T D M A}\left[c_{1}\right]+(1-a) m_{T D M A}\left[c_{2}\right]$ for any two GF-PDFs $c_{1}$ and $c_{2}$ and $0 \leq a \leq 1$. With the above definition of the kernel function, the integral transform, in the following referred to as TDMA forward function, can be written as:

$$
m_{T D M A}\left(g_{\text {set }}\right)=N_{\text {inlet }} \int_{0}^{\infty} c(\hat{g}) K_{\text {TDMA }}\left(g_{\text {set }}, \hat{g}\right) d \hat{g}
$$


Strictly, the kernel function depends on the number of charges on the particles and the total TDMA response is a superposition of the contributions from all charges as detailed in Appendix B.1, Eqs. B.1, B.2 and B.3. Again, the TDMA's forward function (Eq. 2) describes the physics of the TDMA instrument, that is how to calculate the MDF from a given GF-PDF. However, the primary aim of TDMA applications is to determine the GF-PDF of particles exposed to a defined treatment from the observed MDF. This involves solving the inverse problem of Eq. 2.

Three hypothetical GF-PDFs along with the corresponding calculated MDFs are shown in Fig. 3. The GF-PDFs shown are a Gaussian, a triangle and a rectangle, all having a mean GF of 1.4 and a standard deviation of 0.06 . Fig. 3B illustrates that retrieving the GF-PDF from the MDF is an ill-posed inversion problem due to the relatively large width of the kernel function. It is impossible to recover the fine details of the shape of the GF-PDF, always bearing in mind that only a limited number of points of the MDF tainted with uncertainties can be obtained by a measurement. However, the three GF-PDFs shown in Fig. 3B are not so different as they might appear at a first glance. The slopes of the corresponding GF-CDFs (cumulative distribution function; Fig. 3A) are similar at any GF, thus showing that the differences between the three distributions in number fraction of particles found across GF-ranges of finite width are only small.

The underlying principle of TDMA inversion approaches is to find an inverted GF-PDF such that a minimum $\chi^{2}$-residual is obtained between the measured MDF and the reconstituted MDF (R-MDF), which can be calculated from the inverted GF-PDF using the TDMA's forward function. Some sort of an a priori assumption on the properties of the GF-PDF such as e.g. its shape or smoothness is required for any inversion approach. The assumption made in Stolzenburg's TDMAfit algorithm (Stolzenburg and McMurry, 1988) is to describe the GF-PDF as a superposition of multiple Gaussians. In this study we introduce a new approach, TDMAinv, in which the inverted GF-PDF is described as a piecewise linear function at predefined growth factor values as illustrated in Fig. 4. The grey line on panel A represents a simulated MDF, which was calculated from a hypothetical true GF-PDF (shown as grey lines on panels $\mathrm{B}, \mathrm{C}$ and D) using the TDMA forward function. The thick black line on panel B is the inverted GF-PDF obtained with TDMAinv from the simulated measurement, and the black line on panel $\mathrm{A}$ is the corresponding R-MDF.

TDMAinv minimises the $\chi^{2}$-residual between the R-MDF and the measured (simulated) MDF by fitting the values of the piecewise linear GF-PDF at the predefined growth factor values accordingly. The growth factor values are kept fixed during fitting (i.e. $0.95,1.05, \ldots$ in the example shown in Fig. 4B), and the inverse values of the estimated measurement uncertainties 
(Sect. 2.3) are thereby used as weighting factors to determine the $\chi^{2}$-residual. Additionally the physical constraint that the GF-PDF must be $\geq 0$ for all GFs is applied upon minimisation of the $\chi^{2}$-residual in order to limit oscillations occurring at too high resolution of the inversion (see Sect. 3.1). The TDMAinv code is implemented in Wave Metrics' Igor Pro software, which uses the Levenberg-Marquardt algorithm to search the fit parameters that minimise the $\chi^{2}$-residual. The Igor code is available on request (http://people.web.psi.ch/gysel/). There are discernible shape differences remaining between the true and the inverted GF-PDF, even though the inversion approach achieves minuscule differences between the MDF and the R-MDF. This reinforces the above statement that it is impossible to retrieve the fine details of the GF-PDF, independent of the inversion approach. The thin black line in Fig. 4B shows the result of an alternative variant of TDMAinv, where the inverted GF-PDF is described as a piecewise constant function instead of a piecewise linear function. The corresponding R-MDF is not shown on panel A because it is not distinguishable by eye from the black R-MDF. The shapes of inverted GF-PDFs obtained with the two alternative variants of TDMAinv agree within uncertainty. The two variants are hence equivalent though the piecewise linear is preferred over the piecewise constant, when it comes to visualisation. In two-dimensional image plots showing a time series of inverted GF-PDFs, the colour scale changes are gradual in the former case, which is a much better guide to the eye compared to the latter choice, where the changes are incremental and always exactly at the boundaries of the predefined bins.

The kernel function is a key element for the inversion of TDMA data. The effective width of the kernel function of a TDMA instrument can be determined according to the methodology described in Appendix B.2. Fig. 5 illustrates that a narrow monomodal (black shading) and a bimodal (grey shading) GFPDF with separated modes, can result in similar MDFs (black diamonds and grey circles, respectively), if the former is measured with a TDMA having a broader kernel function (e.g. larger aerosol : sheath flow ratio). This ambiguity has consequences for the inversion of observed MDFs. Inverting any of these two MDFs will result in a bimodal GF-PDF if a narrow kernel function is used, while using a broad kernel will result in narrow monomodal GF-PDF. Generally, using a too narrow kernel may provide apparent structure in the GF-PDF that is not actually present, whereas using a too broad kernel may merge nearby separated modes together into a single narrow mode. Exact knowledge of the kernel function is hence critical when it comes to resolving fine structures of the GF-PDF or detection of external mixtures with a small difference in the modal GF. The actual width of the kernel is also important for quantification of the growth spread factor of mixed aerosols, particularly if the GF spread between individual particles is small. However, using a somewhat too narrow kernel does neither affect the retrieved mean GF nor the growth spread factor reported for mixed aerosols with a relatively large growth spread factor. 


\subsection{Measurement uncertainty}

The number of counts $n$ in a data point of the MDF is affected by uncertainties. Statistical limitations associated with low counts are unavoidable, resulting in an uncertainty of $\Delta n_{\text {poiss }}=\sqrt{n}$. Variations of the aerosol concentration at the TDMA inlet also result in uncertainties, which can be captured with an error proportional to $n$ : $\Delta n_{\text {inlet }}=k_{\text {inlet }} n$, where $k_{\text {inlet }}$ is usually $\sim 5 \%$ for ambient measurements and $\sim 5-10 \%$ behind aerosol generators (Appendix B.2.3). We also add $\Delta n_{\text {kernel }}=k_{\text {kernel }} n_{\text {max }}$ to account for uncertainties in the exact shape of the TDMA kernel, where $n_{\max }$ is the maximum number of counts in any data point of the MDF. A typical value for $k_{\text {kernel }}$ is $1 \%$. The combined estimated uncertainty becomes then $\Delta n_{\text {overall }}=\sqrt{n_{\text {poiss }}^{2}+n_{\text {inlet }}^{2}+n_{\text {kernel }}^{2}}$, which is used on a point by point basis for calculating the $\chi^{2}$-residual of the R-MDF against the measured MDF. Comparison of MDFs from offset calibrations with the TDMA kernel function allows judging whether the error estimate $\Delta n_{\text {overall }}$ is adequate (Appendix B.2.3). Otherwise $k_{\text {inlet }}$ has to be adapted accordingly.

Knowledge of the counting uncertainty $\Delta n_{\text {overall }}$ is required for appropriate weighting of each data point of the MDF during data inversion (Sect. 2.2) as well as for quantitative interpretation of the $\chi^{2}$-residuals of the inversion results (Sect. 3.1). TDMA measurements are also tainted with random and systematic measurement uncertainties in $\mathrm{RH}$ and sizing and they may be affected by shape effects in the case of non-spheric particles. The uncertainty solely associated with the shape of the kernel is still typically $\sim 1 \%$ and, though the abovementioned random and systematic errors may be larger, they are treated independently of the data inversion.

\subsection{Interpretation of inverted GF-PDF}

The primary data returned by TDMAinv when using the piecewise linear approach is a list of values of the inverted GF-PDF at the predefined growth factor values, thus defining the inverted GF-PDF as a piecewise linear function. The integral area of the inverted GF-PDF is by definition normalised to unity. The number and volume weighted mean GF are naturally defined as the $1^{\text {st }}$ - and $3^{r d}$-moment mean values of the GF-PDF, respectively. The growth dispersion factor or growth spread factor according to the definition by Stolzenburg and McMurry (1988) is defined as standard deviation of the GF-PDF divided by the mean GF, respectively. Integration of the GF-PDF between two GFs delivers the number fraction of particles exhibiting a GF within this range. In the case of a multimodal GF-PDF with separated modes, the mean GF and the growth spread factor of an individual mode are obtained by evaluating the GF-PDF just across the GF range covered by this mode. 
Such information is equivalent to the number fraction, mean GF and growth spread factor of the Gaussian modes delivered by the multimodal TDMAfit algorithm, as detailed in Sect. 3.2. Explicit equations for the above terms are provided in Appendix C.

\subsection{Correction for $R H$ variations about target value}

In field studies a HTDMA is often operated at a constant $\mathrm{RH}$ with typical target values of $R H_{\text {target }}=90 \%$ or $85 \%$. It is sometimes difficult to keep the relevant $\mathrm{RH}$ in the HTDMA perfectly constant and on target. Typically all data measured within a $\mathrm{RH}$ tolerance of e.g. $\Delta R H= \pm 2 \%$ are reported. In such cases we recommend to apply an empirical correction to the measured GFs and GF-PDFs in order to reduce associated uncertainties. A GF, $g_{\text {meas }}$, measured at relative humidity $R H_{\text {meas }}$ can be corrected in good approximation to a target relative humidity $R H_{\text {target }}$ using single-parameter growth parameterisations such as

$$
\begin{aligned}
& g_{\text {corr }}=\left(1+\left(g_{\text {meas }}^{3}-1\right) \frac{\left(1-R H_{\text {meas }}\right) R H_{\text {target }}}{\left(1-R H_{\text {target }}\right) R H_{\text {meas }}}\right)^{1 / 3} \\
& g_{\text {corr }}=\left(1-R H_{\text {target }}\right) \frac{\ln \left(g_{\text {meas }}\right)}{\ln \left(1-R H_{\text {meas }}\right)} \\
& g_{\text {corr }}=\left(\frac{g_{\text {meas }}^{3}-1}{\left(g_{\text {ref }}\left(R H_{\text {meas }}\right)\right)^{3}-1}\left(\left(g_{\text {ref }}\left(R H_{\text {target }}\right)\right)^{3}-1\right)+1\right)^{1 / 3}
\end{aligned}
$$

where $g_{\text {corr }}$ is the corrected GF. Eq. 3 is based on the hygroscopic growth parameterisation used in Dick et al. (2000), with the coefficients $b$ and $c$ in their Eq. 1 set to zero. Eq. 4 uses the $\gamma$-model, $g=(1-R H)^{-\gamma}$, based on Eq. 18 in Keith and Arons (1954). Eq. 5 is based on the concept of apparent soluble volume fraction introduced by Pitchford and McMurry (1994), where $g_{r e f}(R H)$ describes the hygroscopic growth curve of a reference substance in pure form (typically ammonium sulphate). Fig. 6A illustrates the principle of the $\mathrm{RH}$ correction with the example of pure ammonium sulphate particles. The big black circle represents the GF that would be measured if the relevant $\mathrm{RH}$ was exactly at the target value of $90 \%$. The thick black error bar indicates the GF uncertainty corresponding to a $\mathrm{RH}$ measurement accuracy of $\pm 1 \% \mathrm{RH}$. Smaller or larger GFs (open squares) would be measured if the RH in DMA2 was $88 \%$ or $92 \%$, respectively. Reporting GFs measured between $88-92 \% \mathrm{RH}$ at $\mathrm{RH}=90 \%$ without correcting the GFs for the $\mathrm{RH}$ offset from the target value, i.e. following the black arrows from open squares to small solid squares in Fig. 6A, results in substantially increased measurement uncertainty (thin black error bar) because of contributions from both $\mathrm{RH}$ measurement accuracy 
and offset of the RH from the target value. Alternatively, GFs measured at an $\mathrm{RH}$ slightly off target can be corrected to the target RH by applying the above empirical correction (Eq. 3, 4 or 5), i.e. following the grey arrows from open squares to the grey circles in Fig. 6A, before reporting the corrected GFs at the target $\mathrm{RH}$. The resulting combined measurement uncertainty (grey error bar) is just slightly larger than for a measurement without an $\mathrm{RH}$ offset, as long as the gradients of the actual growth curve (thick black line) and the empirical growth curve (dotted grey lines) used for the correction are similar. A reduction of the measurement uncertainty is always achieved by applying this correction, as long as the local gradient of the empirical growth model is smaller than twice the actual gradient of the particle's growth curve. The latter condition is easily fulfilled for any internally-mixed aerosol as long as the accepted $\mathrm{RH}$ band is sufficiently narrow and neither deliquescence nor efflorescence transitions occur within this RH band.

The correction becomes somewhat more involved for quasi-internally or externallymixed aerosols, where individual particles exhibit different GFs. In such cases the corrected GF-PDF, $c_{\text {corr }}\left(g_{\text {corr }}\right)$, becomes:

$$
c_{\text {corr }}\left(g_{\text {corr }}\right)=c_{\text {meas }}\left(g_{\text {meas }}\right)\left(\frac{\partial f}{\partial g_{\text {meas }}}\left(g_{\text {meas }}, R H_{\text {meas }}, R H_{\text {target }}\right)\right)^{-1}
$$

where $f$ is an empirical function such as Eq. 3, 4 or 5 to correct the GFs for the $\mathrm{RH}$ difference: $g_{\text {corr }}=f\left(g_{\text {meas }}, R H_{\text {meas }}, R H_{\text {target }}\right)$. Fig. $6 \mathrm{~B}$ illustrates the correction principle for a GF-PDF obtained from a measurement at $92 \%$ $\mathrm{RH}$, while the target $\mathrm{RH}$ was $90 \%$. The black crossed line shows a synthetic example GF-PDF at $92 \% \mathrm{RH}, c_{\text {meas }}$. The solid black line shows the corresponding GF-PDF, $c_{\text {nocorr }}$, if it is reported at $90 \% \mathrm{RH}$ without applying any correction, i.e. just following the horizontal black arrows. Let us now focus on the point $c_{\text {meas }}(1.55)=2.77$ at $\mathrm{RH}=92 \%$ (solid black circle) of the measured GF-PDF. It remains naturally unchanged when no correction is applied, i.e. $c_{\text {nocorr }}(1.55)=2.77$ at $\mathrm{RH}=90 \%$ (open black circle). Applying the above empirical correction (Eq. 6) delivers the RH corrected GF-PDF ( $c_{\text {corr }}$; grey line). The grey arrow starting at the solid black circle shows the effect of the correction: $g_{\text {meas }}=1.55$ at $\mathrm{RH}=92 \%$ becomes $g_{\text {corr }}=1.46$ at $\mathrm{RH}=90 \%$ (black and open grey squares, respectively), and the corresponding value of the GF-PDF increases from $c_{\text {meas }}(1.55)=2.77$ at $\mathrm{RH}=92 \%$ to $c_{\text {corr }}(1.46)=3.16$ at $\mathrm{RH}=90 \%$ (solid black and open grey circles, respectively). This increase of the values of the GF-PDF is caused by the compressing effect of the $\mathrm{RH}$ correction (if $\left.R H_{\text {meas }}>R H_{\text {target }}\right)$. The GF-PDF gets compressed because the gradient of the empirical growth curves used for the RH correction decreases with decreasing GF (see grey dotted lines and grey arrows). This is also nicely seen when comparing the black and grey lines. 


\section{Results}

\subsection{Proof of concept}

Here we show that the TDMAinv data analysis algorithm introduced in Sect. 2.2 fulfils the specifications listed in Table 1. For this purpose, a true GF-PDF, representing an externally-mixed aerosol with three distinct modes, was constructed (grey line in Fig. 4B, C and D). The corresponding MDF was calculated using the TDMA forward function, and 100 copies of the MDF with superposed measurement noise were generated. The noise was simulated according to Sect. 2.3 thus accounting for counting statistics $\left(\Delta n_{\text {poiss }}\right)$ and potential variations of the particle concentration at the TDMA inlet $\left(\Delta n_{\text {inlet }}\right.$ with $k_{\text {inlet }}=5 \%$ ). TDMAinv was then applied to invert simulated noisy MDFs. Table 2 summarises key integral properties of the resulting inverted GF-PDFs for different inversion settings. The columns show the mean GF of the inverted GF-PDF and the number fractions of particles with GFs $g<1.2,1.2<g<1.435$, and $g>1.435$. The values of the true GF-PDF are shown in the second row, while the third row ('PWL', $\Delta \mathrm{GF}=0.10$ ) contains the reference piecewise linear (PWL) inversion with appropriately chosen resolution (thick black line in Fig. 4B). This reference inversion returns the mean GF within less than $1 \%$ of the correct value, and also the number fractions of particles in different GF ranges are accurately determined. Using different predefined growth factor values shifted by half a bin width ('PWL shifted', $\Delta \mathrm{GF}=0.10$ ) does not change the outcome of the inversion beyond uncertainty, and the same is also true when the inverted GF-PDF is represented as a piecewise constant ('PWC') instead of a piecewise linear function. Using a kernel calculated with $10 \%$ too narrow $\mathrm{FWHM}_{D}$ of the DMA transfer functions does not affect the outcome either ('PWL narrow kernel'). Increasing the resolution ('PWL', $\Delta \mathrm{GF}=0.05$ ) causes unreal oscillations in the inverted GF-PDF (Fig. 4C). However, other than that the results of the inversion are still accurate. Decreasing the resolution ('PWL', $\Delta \mathrm{GF}=0.20$ and 'PWL shifted', $\Delta \mathrm{GF}=0.20$ ) results in smoothing errors because there are not enough free parameters available to reproduce the complex shape of the true GF-PDF (Fig. 4C). The retrieved mean GF is still accurate, whereas the uncertainty of the number fractions of particles in different GF ranges increases and changes when using different predefined growth factor values. The row 'noisy MDF' shows what is obtained when the MDF is directly used to determine the integrated parameters of the GF-PDF without applying any data inversion. This approximation results in a systematic overestimation of the mean GF and of the number fraction of particles with a GF above the mean GF on the one hand, and an underestimation of the number fraction of particles with a GF below the mean GF on the other hand. These systematic biases occur because the TDMA transfer probability increases with increasing GF of the particles (Fig. 2). 
The piecewise linear TDMAinv inversion is robust regarding the retrieval of the mean GF or other integral properties of the GF-PDF determined for a wide GF range. However, choosing an appropriate resolution is critical when it comes to accurate determination of fine structures of the GF-PDF such as the number fractions of particles in narrow GF ranges. Normally the correct outcome of the inversion is not known and therefore an objective tool to find the appropriate resolution for a given MDF is required. It has to be stressed that the adequate resolution depends on both the measurement uncertainties as well as the shape of the MDF. Higher resolution is required for largely internally-mixed aerosols with a small growth spread factor than for largely externally-mixed aerosols. The last column of Table 2 shows the mean and standard deviation of the normalised $\chi^{2}$-residuals $\left(\chi^{2}\right.$ divided by the number of data points) obtained by inverting the set of 100 simulated noisy MDFs. The simulated 'noisy MDF' themselves have $\chi^{2}$-residuals in the order of 1 , confirming that simulating the noise and calculating the $\chi^{2}$-residuals are consistent. $\chi^{2}$-residuals between $\sim 0.5-2$ achieved by the inversion indicate adequate choice of the resolution. Much smaller $\chi^{2}$-residuals indicate too high resolution, resulting in unreal oscillations of the inverted GF-PDFs (black line in Fig. 4C). The oscillations provide apparent structure in the GF-PDF that is not actually present, but do not otherwise affect the accuracy of the inversion. An exception are unreasonably high resolutions causing excessive oscillations, where the fit algorithm may fail. The black line in Fig. 4D shows an example inversion with all fit constraints turned off, thus being similar to the OEM approach by Cubison et al. (2005). This results in excessive oscillations at high resolution, which propagate beyond the support of the MDF. The $\chi^{2}$-residual of this oscillating GF-PDF is smaller than that of all other inverted GF-PDFs shown in Fig. 4. However it is physically unreasonable. The possibility to apply physical constraints is the major advantage of TDMAinv over the OEM approach.

$\chi^{2}$-residuals much larger than 2 indicate too low resolution, resulting in smoothing errors (thin black line in Fig. 4C). In such a case the fine structures of the GF-PDF are not recovered and the number fractions of particles in different GF ranges become increasingly uncertain. The $\chi^{2}$-residuals depend on correct estimation of the measurement uncertainties, particularly on the variability of the concentration $\Delta n_{\text {inlet }}$ of particles of the selected size at the TDMA inlet (see Sect. 2.3). Hence an objective choice for an adequate resolution can only be made if $\Delta n_{\text {inlet }}$ is quantified from e.g. the offset calibrations. In our field experience $k_{\text {inlet }} \approx 5 \%$ turned out to be a good value for ambient measurements (see Appendix B.2.3). In cases when $\Delta n_{\text {inlet }}$ cannot be quantified it is impossible to determine with certainty to what degree fine structures of the GF-PDF such as the number of distinct modes are real or not, whichever data inversion approach is chosen. An alternative criterium for choosing the resolution is required if the absolute levels of the $\chi^{2}$-residuals are not decisive due to unknown measurement uncertainties. In such cases the inversion reso- 
lution should be increased until only a small reduction of the $\chi^{2}$-residual can be achieved by either shifting the predefined growth factor values by half a bin width or by further increasing the resolution.

A more detailed error analysis should be done before fine structures of the inverted GF-PDF are interpreted. For this purpose the R-MDF corresponding to the inverted GF-PDF is taken, and a large number of copies with simulated measurement noise according to the parameters $k_{\text {inlet }}$ and $k_{\text {kernel }}$ is generated. The error statistics for the GF-PDF and its integrated parameters can then be determined by inversion of all noisy MDFs. The resulting $5^{\text {th }}$ and $95^{\text {th }}$ percentiles have been determined for the above example and are shown as error bars in Fig. 4B,C. The error bars obtained for the retrieval with appropriately chosen resolution (Fig. 4B) show that the minima of the GF-PDF found between 1.15 and 1.35 and between 1.35 and 1.55 are most likely real. Large error bars are obtained for the high resolution inversion (Fig. 4C), confirming that the alternating minima and maxima of the GF-PDF are not significant and just caused by oscillations, as forecast from achieving too low $\chi^{2}$-residuals. Analysis of the $\chi^{2}$-residuals for the low resolution inversion indicated significant smoothing errors, which are reflected in very small, hardly discernible error bars (Fig. 4C). This is because slightly different MDF's cannot be distinguished with too low resolution and thus no variation in the inverted GF-PDFs is seen. In the general case, it is not possible to make statements about the uncertainty of retrieved parameters if the measurement uncertainty is unknown.

\subsection{Comparison of TDMAinv with TDMAfit}

Nearby narrow modes in the GF-PDF are a challenge for TDMA data inversion algorithms. Fig. 7 shows the results from a HTDMA measurement of pure $D_{0}=100 \mathrm{~nm}$ ammonium sulphate particles. The RH of 34-38\% (panel A) was around the efflorescence RH of ammonium sulphate, and the measurement was performed in such a way that dry crystals and metastable solution droplets were simultaneously present. This resulted in two separated narrow growth modes with mean GFs of $\sim 1.0$ and $\sim 1.15$. These two modes are hardly discernible in the MDF, as shown with two selected examples on panels $\mathrm{F}$ and $\mathrm{G}$ (line with markers). The corresponding inverted GF-PDFs obtained by the bimodal Gaussian approach (TDMAfit; grey lines) and the piecewise linear inversion (TDMAinv; black lines) clearly reveal the bimodal growth behaviour. The $\chi^{2}$-residuals achieved by the two inversion approaches are around one (panel B), thus indicating appropriate data inversion. The mean GF (panel C) and the number fraction (panel D) of particles found in the liquid and solid modes of the inverted GF-PDFs were calculated according to the definitions given in Appendix C. Both approaches deliver correct GFs for either mode, when compared with the theoretical reference values (solid and dashed lines 
on panel $\mathrm{C}$ ), and the retrieved number fractions agree well. Only the mean GF of the liquid mode of the leftmost data point is tainted with larger uncertainty because only $\sim 10 \%$ of the particles are in this mode. Small differences between the approaches are found for the retrieved standard deviation $\sigma$ of the two modes (panel E). TDMAinv sometimes delivers somewhat smaller $\sigma$ for the liquid mode, whereas the $\chi^{2}$-residuals at these points are somewhat smaller compared to TDMAfit. This indicates that the GF-PDF obtained with TDMAinv is somewhat more likely to be true. However, these differences reflect the fact that determining the standard deviations of such nearby narrow modes is at the limit of the TDMA's resolution power. In summary both approaches deliver equivalent results. Nevertheless, a major advantage of TDMAinv is that convergence of the fit routine is robust and independent of the initial guess, though adequate resolution must be used to prevent oscillations or smoothing errors as discussed in Sect. 3.1. Test series using initial guesses with random shape and random area have been performed for different MDFs including the examples shown in Figs. 4 and 8. TDMAinv always converged to the solution, with the one reservation that the area of the initial guess is at most ten times the area of the solution. Successful convergence of TDMAfit depends critically on the initial guess. In the above example we had to use the results delivered by TDMAinv as initial guess for the bimodal TDMAfit approach in order to achieve convergence to the reported solution. Generally convergence of TDMAfit becomes more difficult to achieve with increasing number of fitted modes, which has to be done for more asymmetric and/or multimodal GF-PDFs.

\subsection{Sensitivity to counting statistics}

In the following we illustrate the effects of a decreasing total number of counts recorded in the MDF, $n_{\text {total }}$, on the accuracy of the inverted GF-PDF. An example GF-PDF with $g_{\text {mean }}=1.50$ and $\sigma=0.0323$ was sent through the TDMA's forward function to obtain the shape of the corresponding synthetic MDF. The latter was then scaled to different $n_{\text {total }}$ ranging from 10 to 5000. 100 copies with superposed measurement noise were generated of each scaled MDF. The noise was simulated according to Sect. 2.3 thus accounting for counting statistics $\left(\Delta n_{\text {poiss }}\right)$ and potential variations of the particle concentration at the TDMA inlet $\left(\Delta n_{\text {inlet }}\right.$ with $\left.k_{\text {inlet }}=5 \%\right)$. Both TDMAinv and TDMAfit were then applied to invert all noisy MDF's and $g_{\text {mean }}$ as well as $\sigma$ of the retrieved GF-PDFs were determined. The average values with associated standard deviation of $g_{\text {mean }}$ (panel A) and $\sigma$ (panel B) are shown as markers with error bars in Fig. 8, as obtained from the 100 inversion results at each counting level. $g_{\text {mean }}$ and $\sigma$ of the noisy MDFs (no inversion) are also shown. $g_{\text {mean }}$ of the GF-PDF is rather insensitive to small $n_{\text {total }}$ with a $1 \sigma$-accuracy of $\sim \pm 2 \%$, $\pm 1 \%, \pm 0.5 \%$ and $\pm 0.2 \%$ for $n_{\text {total }} \geq 10,20,100$ and 500 , respectively. The 
fact that the variability of $g_{\text {mean }}$ (shown as error bars) is equal for the noisy MDFs and for the inverted GF-PDFs shows that the accuracy of the information is already lost during the (simulated) measurement and not during the inversion. The average values of $g_{\text {mean }}$ of the MDFs and of the inverted GF-PDFs agree too, thus corroborating that the measurement noise does not induce a bias in the inversion results. The $\sigma$ of the GF-PDF is much more sensitive to small $n_{\text {total }}$ with an accuracy of $\sim \pm 80 \%, \pm 50 \%, \pm 10 \%$ and $\pm 5 \%$ for $n_{\text {total }} \geq 10,50,1000$ and 5000, respectively. The $\sigma$ obtained from the MDF without inversion is naturally too large, though its variability again shows that the accuracy of the information is mostly lost during the measurement and not during the inversion. The accuracy limitations provided above are only valid for this particular example GF-PDF along with this magnitude of measurement noise. For example the relative sensitivity of $\sigma$ to small $n_{\text {total }}$ decreases with increasing breadth of the GF-PDF. Unlike in the above example, laboratory or field experiments with a HTDMA often provide just a single or very few measurements of an aerosol sample. Assessment of the potential accuracy/uncertainty of the retrieved parameters needs careful consideration with the error simulation as detailed in the last paragraph of Sect. 3.1.

\subsection{Multiple charges}

In standard operation a bipolar charger is used upstream of the TDMA to bring the sample to charge equilibrium, before singly, doubly, and triply charged particles with respective diameters $D=D_{0}, u_{2}\left(D_{0}\right)$ and $u_{3}\left(D_{0}\right)$ are selected by the first DMA. The number fractions of singly, doubly or triply charged particles behind DMA1 depend both on the size dependent charging probabilities as well as on the shape of the dry particle number size distribution. Often the singly charged particles dominate such that the TDMA forward function simplifies to Eq. B.5 and data analysis is straight forward. In cases when significant fractions of particles with different number of charges are selected by DMA1 - e.g. singly and doubly charged particles - then the measured MDF is a superposition of contributions from different dry sizes (Eq. B.1) and appropriate data inversion is hardly possible. Unfortunately an SMPS-style multicharge correction cannot be applied because the relative contributions from singly and multiply charged particles to every data point of the MDF cannot be distinguished, even if the GF-PDFs $c\left(g, D_{i}=u_{i}\left(D_{0}\right)\right)$ of the larger, multiply charged particles were known.

Accurate data inversion despite multiple charge effects can be possible in special cases, when exclusively doubly or triply charged particles are selected by DMA1. This is illustrated in the following with an example from an $\alpha$ pinene photo-oxidaton experiment in a smogchamber. Fig. 9A shows that the physical diameter of multiply charged particles is larger than the electrical 
mobility diameter set at DMA1. Doubly charged particles with a mobility diameter of $D_{0}=200 \mathrm{~nm}$ have an electrical mobility diameter of $130 \mathrm{~nm}$, as seen from the intersection of the horizontal dashed line with the grey line. Panel B shows the number fraction of singly, doubly and triply charged particles at the exit of DMA1 as a function of the electrical mobility diameter set at DMA1. These values have been calculated from the number size distribution measured by an SMPS, taking charging and transfer probabilities into account. Singly charged particles constitute the dominant fraction only for set diameters larger than $160 \mathrm{~nm}$, whereas doubly charged particles dominate for set diameters of $\sim 110-135 \mathrm{~nm}$. It seems surprising that doubly charged particles dominate at a smaller size than singly charged particles. However, in this particular case the number size distribution (not shown) is centred at a diameter of $D_{0} \approx 190 \mathrm{~nm}$ and very narrow with hardly any particles smaller than 150 or larger than $230 \mathrm{~nm}$, which could show up as singly or doubly charges particles, respectively, when setting DMA1 to a set diameters of e.g. 130 or $200 \mathrm{~nm}$. The dashed lines on panels $\mathrm{A}$ and $\mathrm{B}$ show that the properties of particles with a mobility diameter of $200 \mathrm{~nm}$ can be investigated by setting DMA1 to an electrical mobility diameter of either $200 \mathrm{~nm}$ or alternatively $130 \mathrm{~nm}$, thus exclusively selecting singly or doubly charged particles, respectively. Standard data inversion (Eq. B.5) can be applied in the former case, whereas in the latter case the data analysis needs to be adapted accordingly. The TDMA forward function for doubly charged particles is obtained by using only the second summand of Eq. B.1 with the kernel $K_{T D M A}^{g, 2}\left(g_{\text {set }}, \hat{g}, D_{0}\right)$ for doubly charged particles.

Fig. 9C shows the full kernel $K_{T D M A}^{g, i}\left(g_{\text {set }}, \hat{g}=1.5, D_{0}=200\right)$ and Fig. 9D the corresponding modal GF for multiply charged particles with a true GF of $\hat{g}=1.5$. The dashed lines show that the MDF peaks at GFs of 1.5, 1.45 and 1.42 for singly, doubly, and triply charged particles, respectively. Therefore it is crucial to use the kernel calculated with the appropriate number of charges because the centre of the kernel function at higher charges is systematically offset towards smaller GFs. It is important to note that the area of the kernel function hardly changes with increasing number of charges, such that the number fraction of particles with different number of charges can be directly compared to determine which charge gives the dominant contribution to the MDF. Duplissy et al. (2008) have applied the kernel functions with higher charges to a HTDMA data set from the above-mentioned smogchamber experiment, thus proving that correct GFs can be retrieved by selecting at an electrical mobility diameter, where multiply charged particles dominate. Measurement of GFs through multiply charged particles sometimes allows extending the measurement range of a HTDMA towards larger sizes. Typical examples are smogchamber experiments which produce a very narrow particle number size distribution growing beyond the single charge equivalent size range of the DMAs. 


\section{Conclusions}

In recent years the TDMA technique has become widely used. The need of appropriate data inversion before interpreting and reporting TDMA data is commonly acknowledged as indispensable (Swietlicki et al., 2008). Nevertheless studies reporting TDMA data without adequately inverted data are still occasionally published. Reasons for this neglect may be either avoiding the complexity of developing an inversion algorithm or just out of ignorance. Currently a large HTDMA data set is being acquired at several EUSAAR supersites (European Supersites for Atmospheric Aerosol Research) across Europe as part of the year of aerosol measurements of the EUCAARI project (European Integrated Project on Aerosol Cloud Climate Air Quality Interactions). Inverting all data collected during such permanent measurements with TDMAfit inversion algorithm would be challenging because it may need considerable manual interference in order to find adequate initial guesses for all individual measurements. The OEM approach is a very efficient tool, however, the results may be physically unreasonable if the chosen resolution is too high.

We have developed a new TDMA data inversion algorithm, TDMAinv, which represents the inverted GF-PDF as a piecewise linear function. A major advantage of our algorithm over TDMAfit is its robust convergence, which is independent of the initial guess chosen. This makes it particularly suitable to analyse large TDMA data sets, while retaining simplicity for standard laboratory measurements. Our TDMAinv-toolkit provides largely automated data analysis and inversion routines and the code is available on request (http://people.web.psi.ch/gysel/). In any case future studies reporting TDMA data should only be acceptable for publication if an appropriate inversion algorithm is used. Furthermore, field studies should aim to report the inverted TDMA data in a form retaining as much information on the GF-PDF as possible. However, we have demonstrated that this requires an adequate inversion algorithm, knowledge of the TDMA's actual kernel function, and careful consideration of the limitations imposed by the measurement noise in the recorded MDFs.

\section{Acknowledgements}

We thank for financial support of this work by the Swiss National Science Foundation, the Swiss GAW programme and the EC project EUSAAR (Contract RII3-CT-2006-026140). Particular thanks to Jonathan Duplissy for his valuable feedback, which helped to improve the functionality of the TDMAinv algorithm and to Ernest Weingartner for helpful discussions and his comments on the manuscript. 


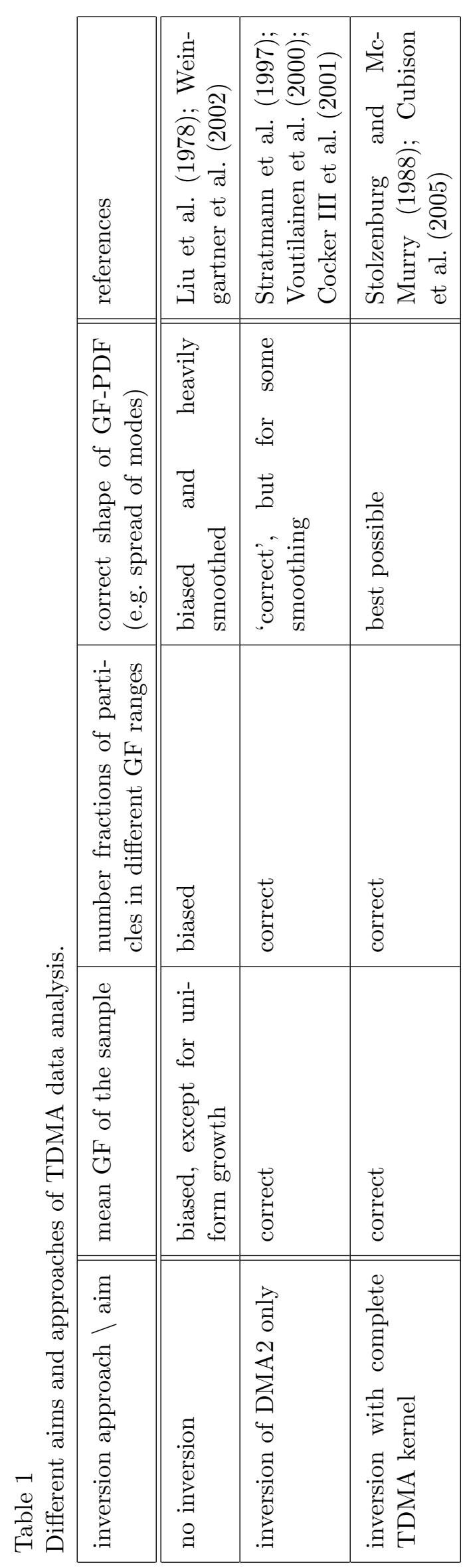




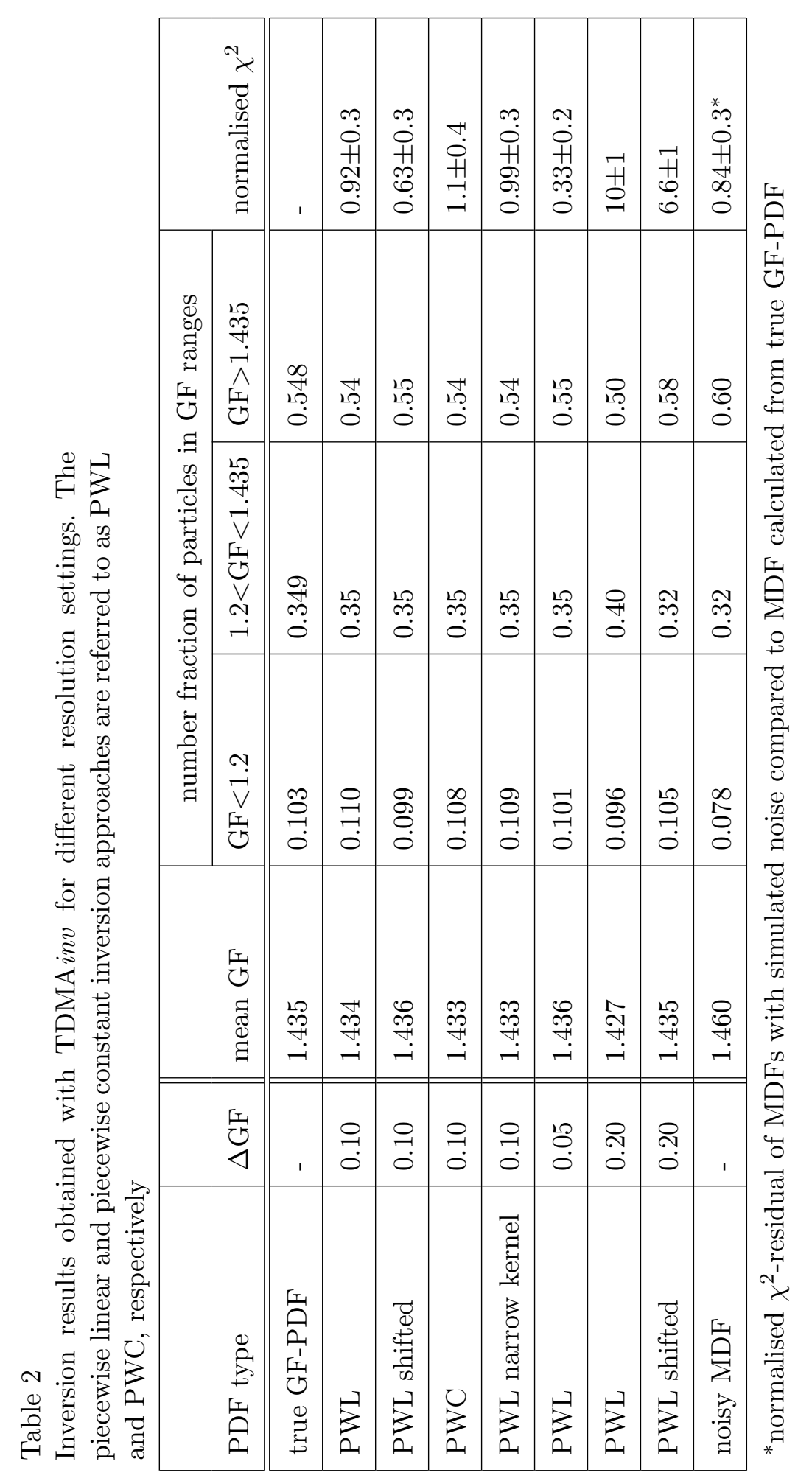




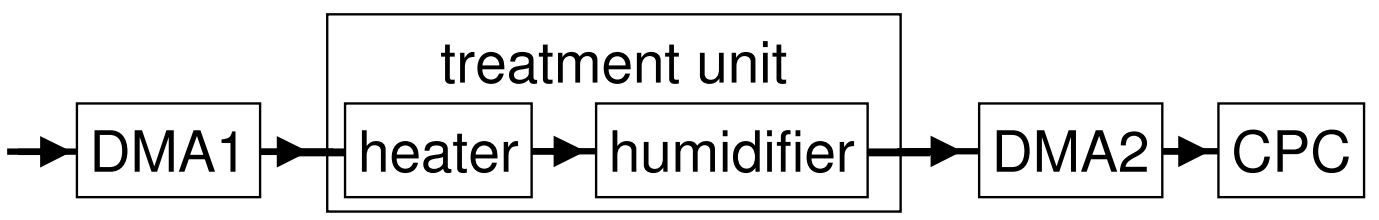

Fig. 1. TDMA set up; this sketch shows the volatilisation-humidification branch of a VHTDMA, in which the treatment unit consists of a thermodesorber and humidifier in series.

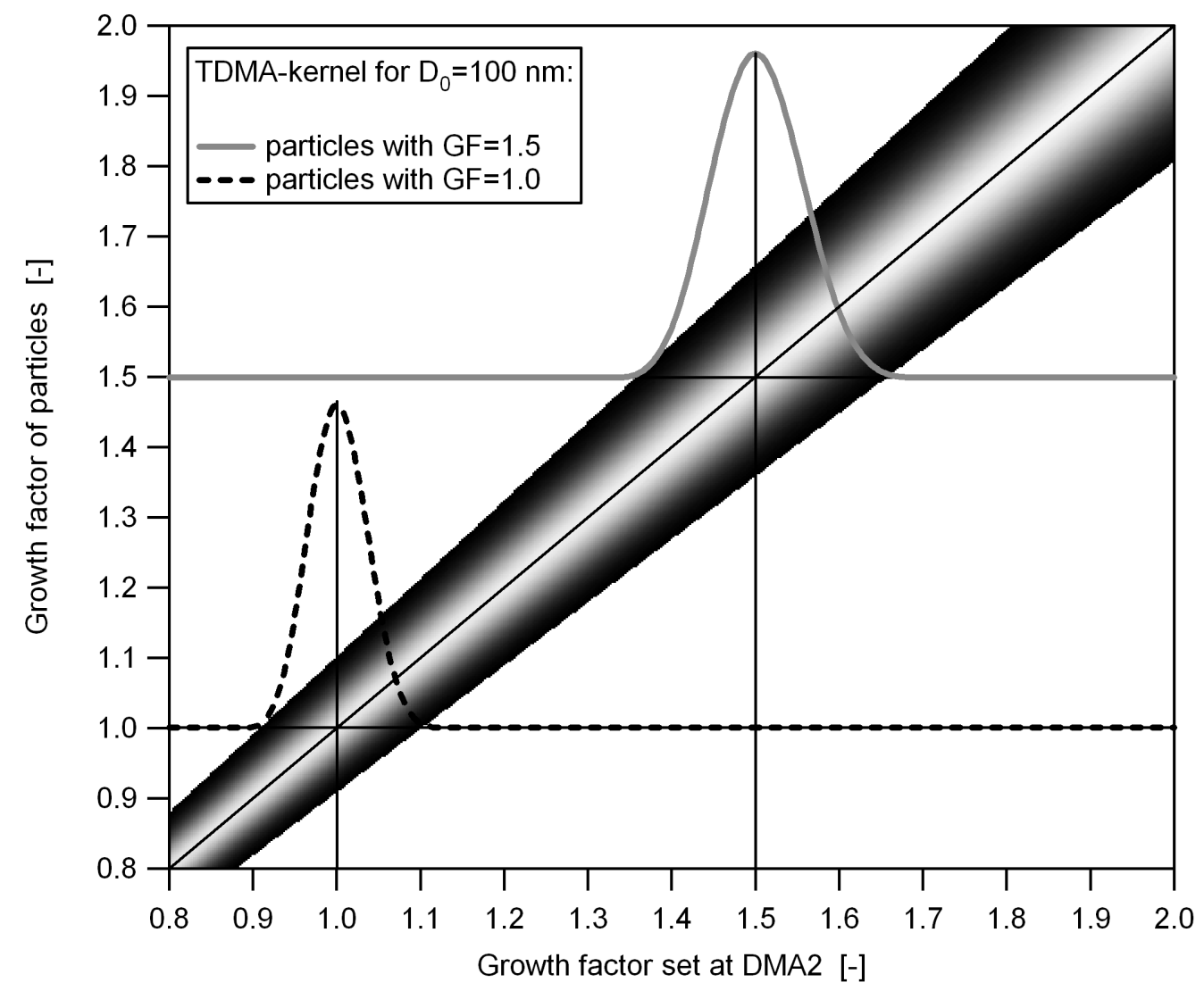

Fig. 2. Image of theoretical TDMA kernel function for an aerosol to sheath flow ratio of 1:10 in both DMAs and for particles carrying a single charge with dry diameter $D_{0}=100 \mathrm{~nm}$. The dashed and grey lines illustrate the TDMA response on particles exhibiting a GF of 1.0 and 1.5, respectively. 


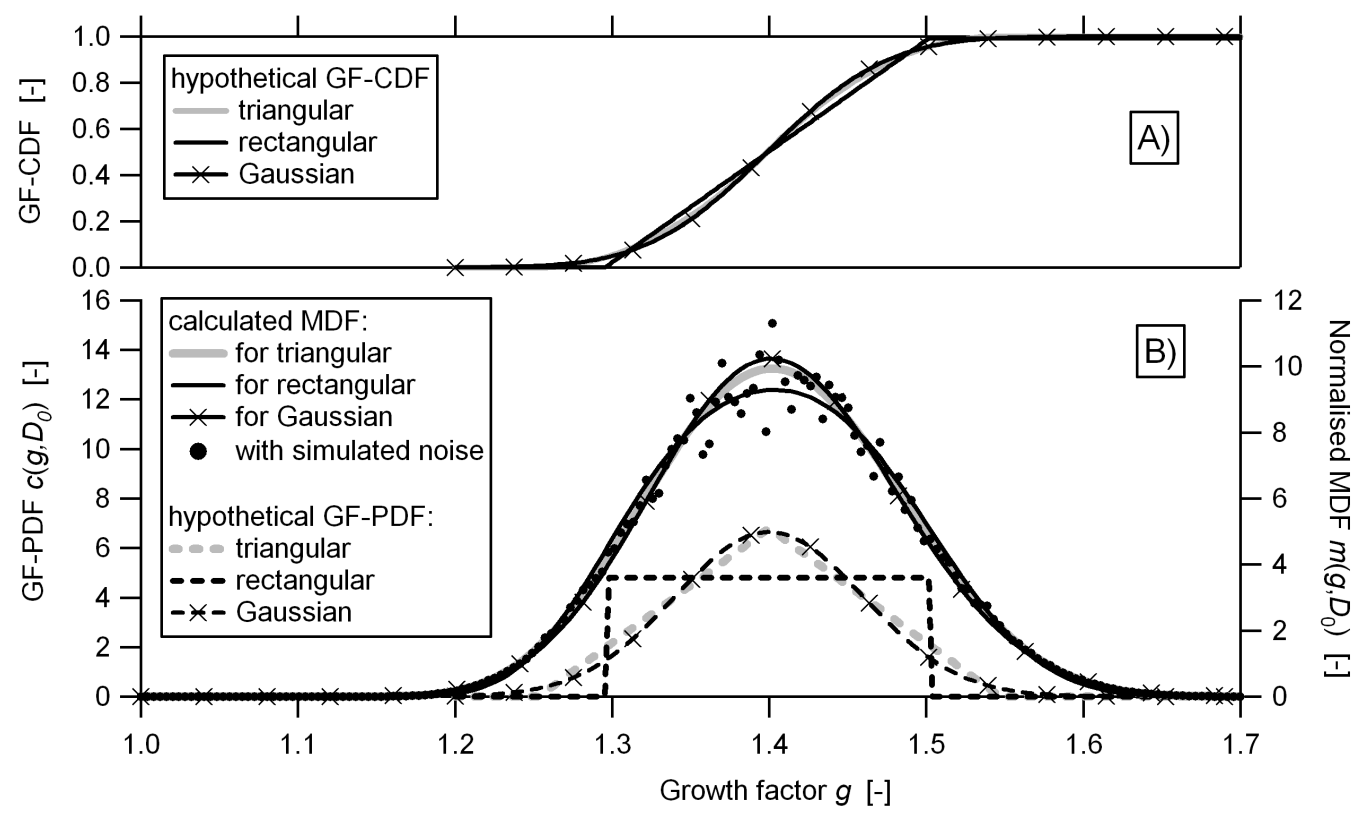

Fig. 3. Sensitivity of the TDMA measurement to minor shape differences of the GF-PDF. The dashed lines on panel B represent three hypothetical GF-PDFs, all with a mean GF of 1.4 and a standard deviation of 0.06 . The corresponding GF-CDFs are shown on panel A. The solid lines on panel B show the TDMA measurement distribution functions (MDF) calculated for these hypothetical GF-PDFs. The solid circles show a synthetic example of a real measurement including noise. 


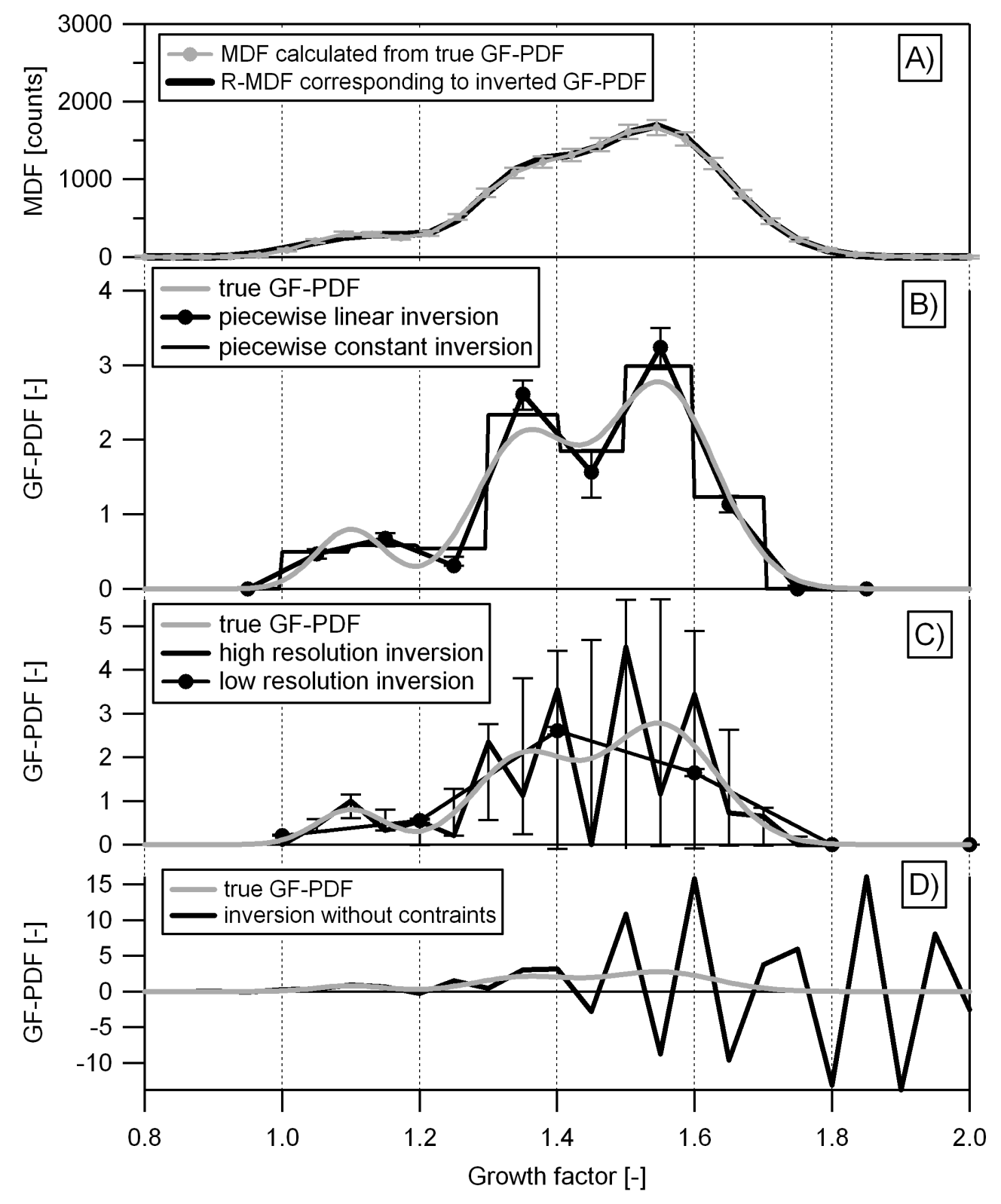

Fig. 4. Principle of TDMAinv. The grey lines on panels B, C, and D indicate an arbitrary true GF-PDF. The corresponding calculated MDF is shown as grey line on panel A. The calculated MDF was inverted representing the GF-PDF as a piecewise linear and piecewise constant function (thick and and thin black lines on panel B, respectively). The black line on panel A shows the R-MDF as obtained by sending the piecewise linear inversion back through the forward function. The effects of choosing too high or too low bin resolution are shown on panel $\mathrm{C}$ as thick and thin black lines, respectively. The black line on panel D illustrates how excessive oscillations extending beyond the support of the MDF can occur if no fit constraints are applied. 


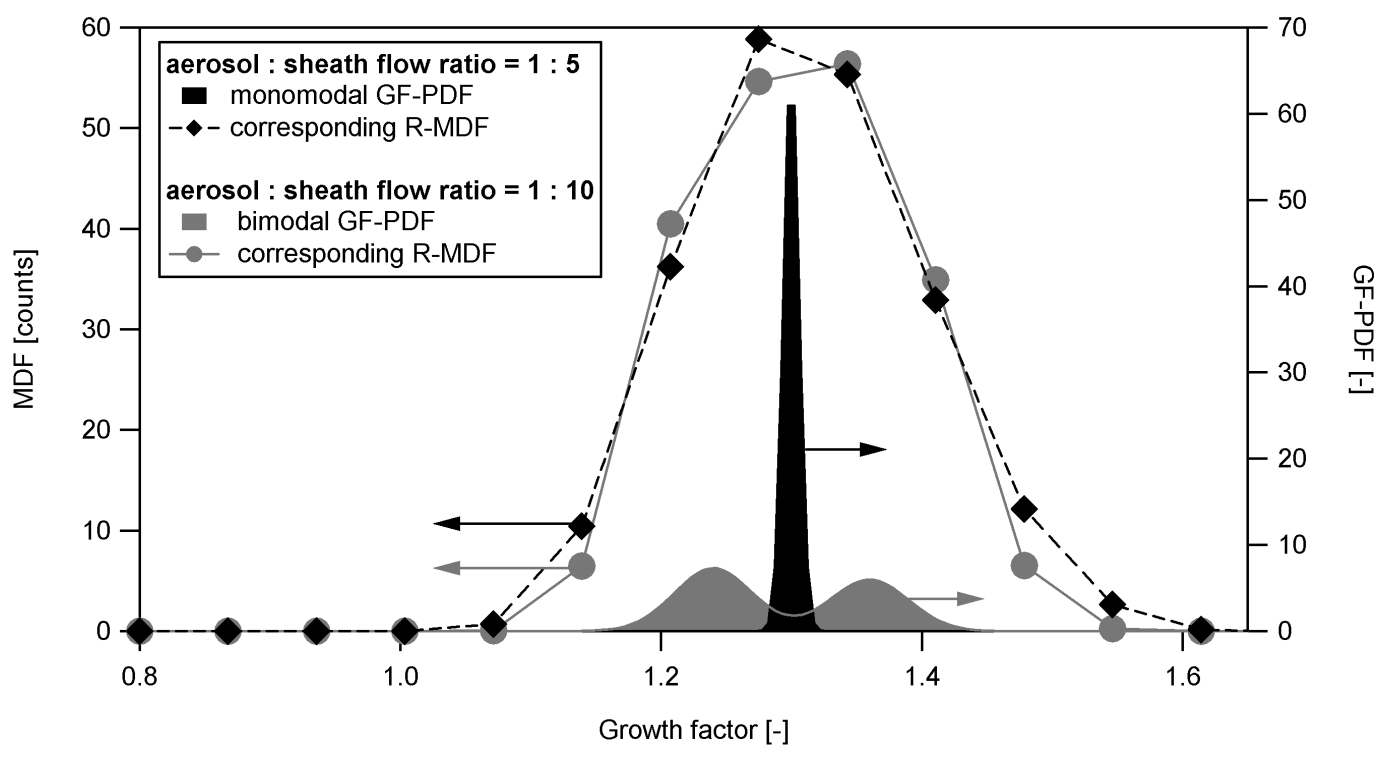

Fig. 5. The black shading and black diamonds show a hypothetical monomodal GF-PDF and the corresponding calculated MDF, respectively, assuming a broad TDMA kernel (aerosol : sheath flow ratio $=1: 5$ ). The grey shading and grey circles show a hypothetical bimodal GF-PDF and the corresponding calculated MDF, respectively, assuming a narrow TDMA kernel (aerosol : sheath flow ratio $=1: 10$ ). The two MDFs are hardly discernible because the differences of the GF-PDF are just compensated by the differences of the kernel function. This ambiguity shows that exact knowledge of the kernel function is critical when it comes to resolving fine structures of the GF-PDF by inversion of an observed MDF. 

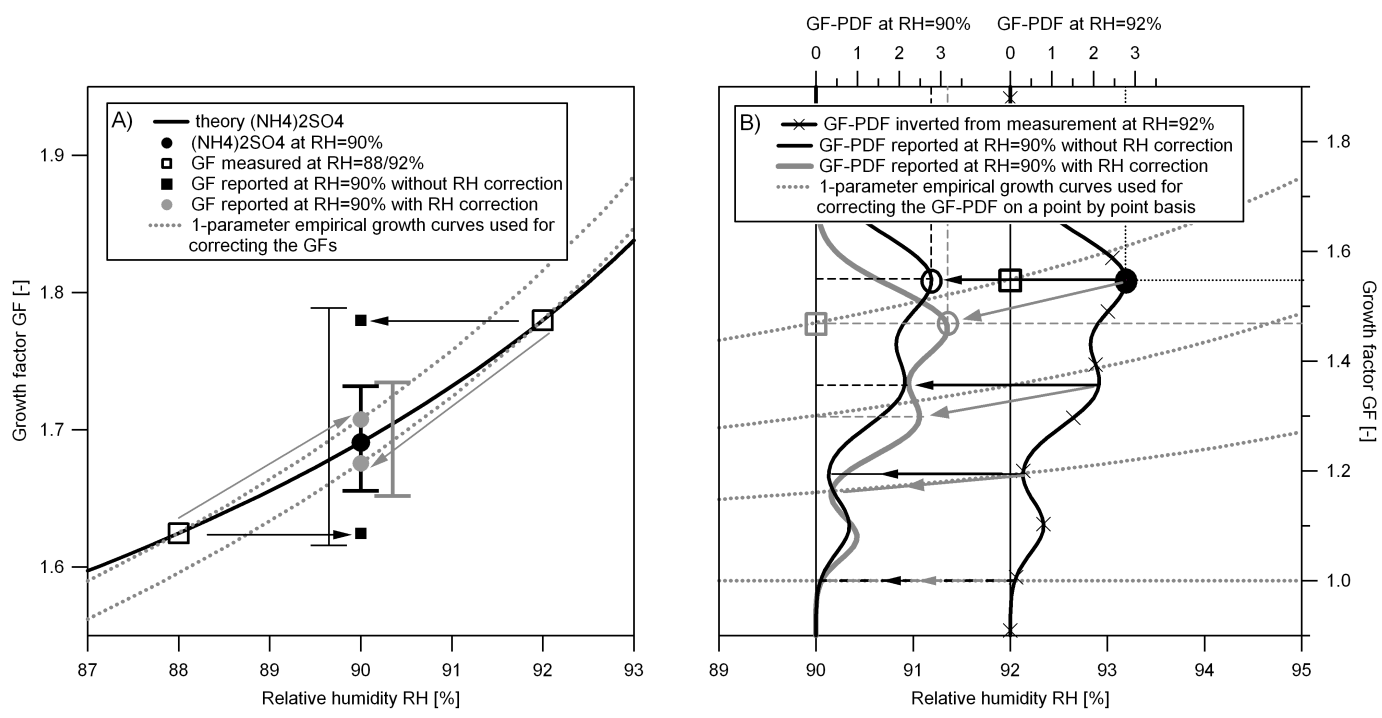

Fig. 6. Correction of hygroscopic GF measurements to target $\mathrm{RH}=90 \%$. A) Internally-mixed sample with a well defined GF. Open squares show the GF obtained from a measurement at $\mathrm{RH}=88 \% / 92 \%$, while grey circles and solid squares show the corresponding $\mathrm{GF}$ reported at target $\mathrm{RH}=90 \%$ with and without applying a $\mathrm{RH}$ correction, respectively. B) Externally-mixed sample with a broad GF-PDF. The crossed black line shows the GF-PDF obtained from a measurement at $\mathrm{RH}=92 \%$, while the grey and black lines show the corresponding GF-PDF reported at target $\mathrm{RH}=90 \%$ with and without applying a $\mathrm{RH}$ correction, respectively. The squares and circles on panel B indicate how the point $c_{\text {meas }}(g=1.55, R H=92 \%)=2.77$ of the GF-PDF changes to $c_{\text {corr }}(g=1.46, R H=90 \%)=3.16$ upon $\mathrm{RH}$ correction. The dotted lines on either panel show growth curves of the empirical 1-parameter model, which was used for the RH correction. 

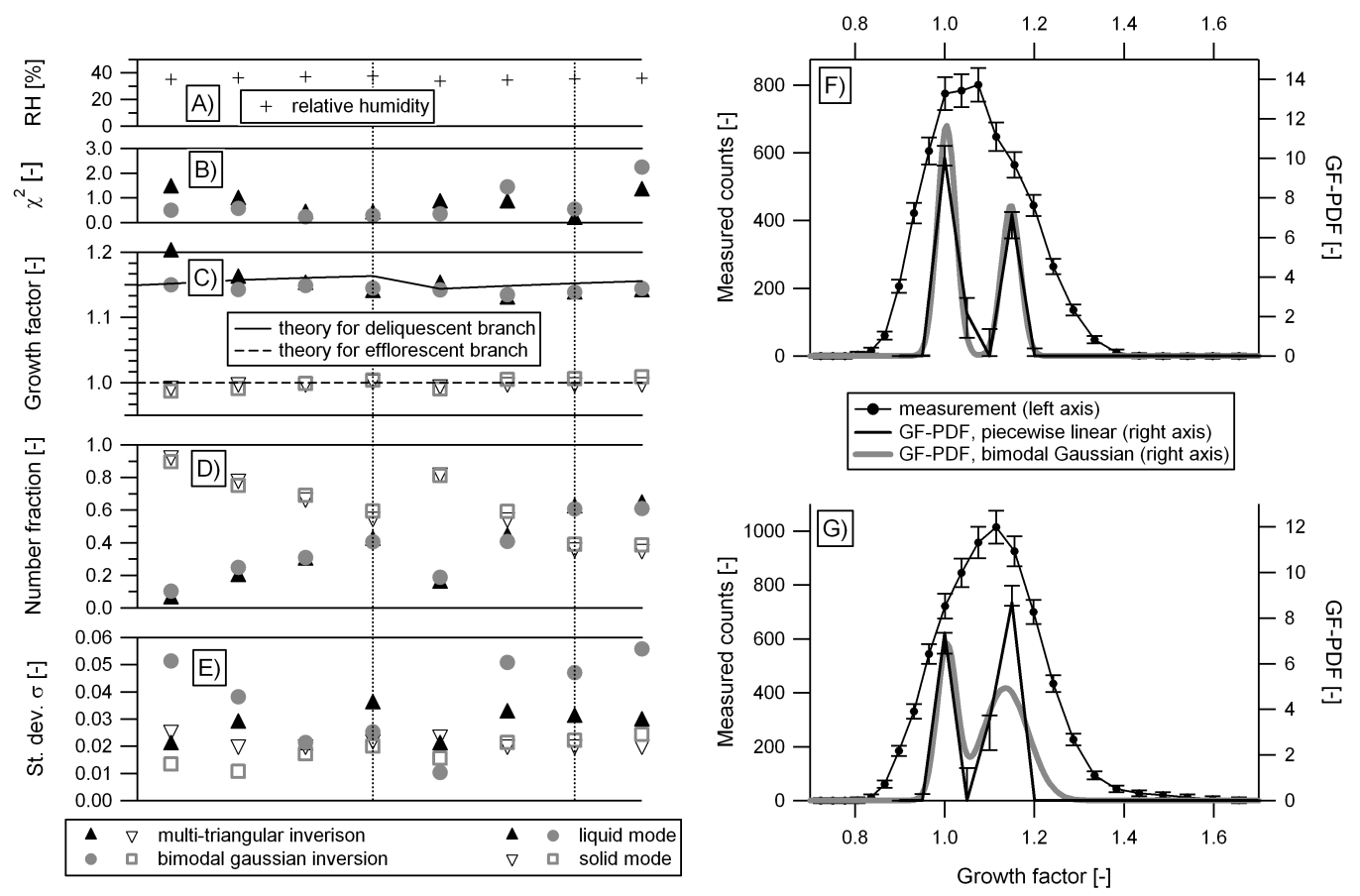

Fig. 7. Comparison of TDMAinv (up/down triangles) with TDMAfit (circles/squares) for bimodal GF-PDFs with two nearby narrow modes. A) relative humidity, B) $\chi^{2}$-residuals achieved by the two inversion approaches, C-E) inversion results for the liquid (up triangles/circles) and solid modes (down triangles/squares) of the retrieved GF-PDF, and F-G) two selected example MDFs (line with markers) along with corresponding inverted GF-PDFs (black/grey lines). 

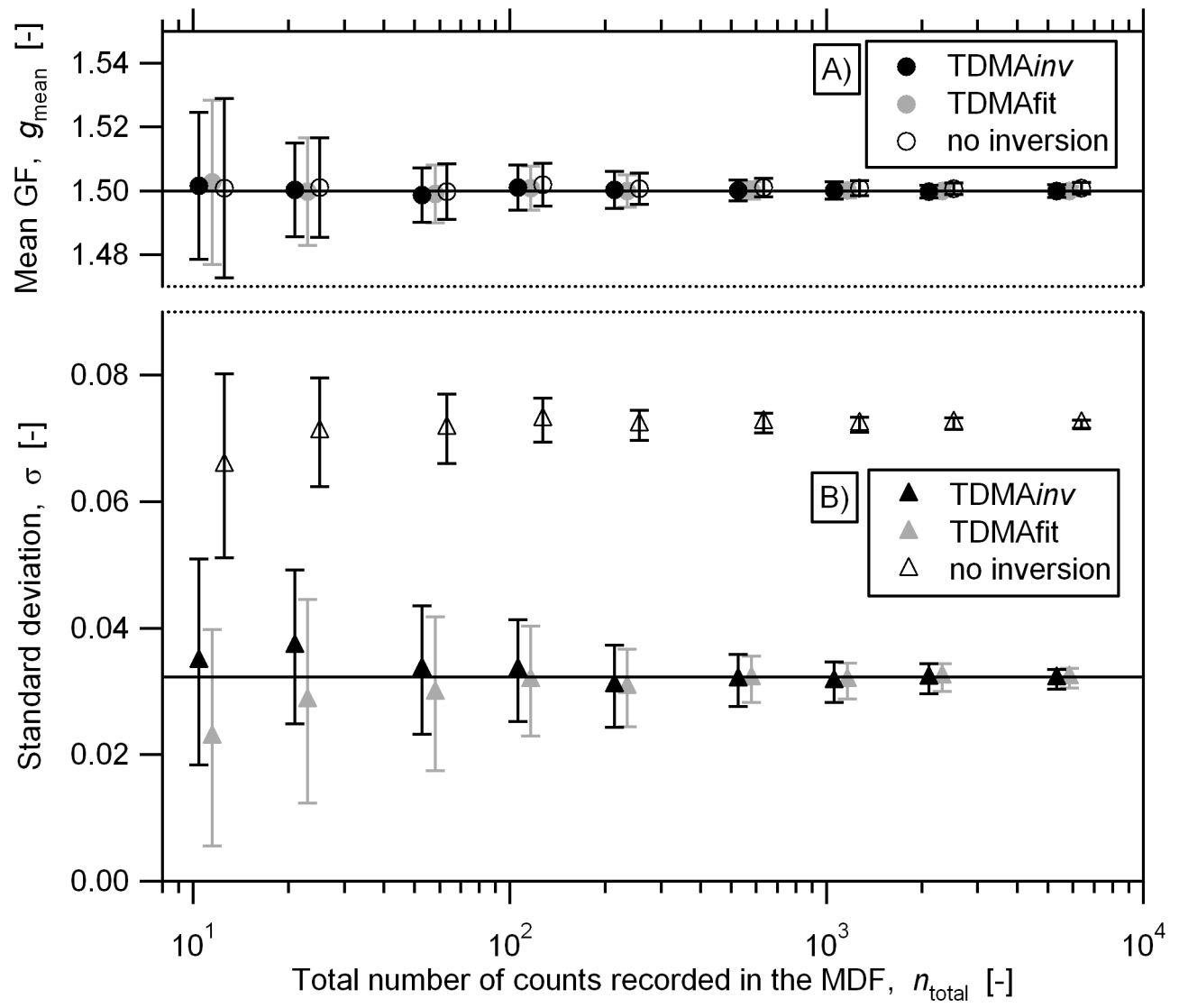

Fig. 8. Sensitivity of A) $g_{\text {mean }}$ and B) $\sigma$ of the GF-PDF to $n_{\text {total }}$ for an example GF-PDF with $g_{\text {mean }}=1.50$ and $\sigma=0.0323$. Each data point was obtained by analysing 100 copies of synthetic MDFs with simulated measurement noise. The average values and associated standard deviation of the inversion results are shown as markers with error bars. $g_{\text {mean }}$ and $\sigma$ of the MDFs (no inversion) are also shown. Note, the grey (TDMAfit) and open (no inversion) markers are plotted with a small horizontal offset. 


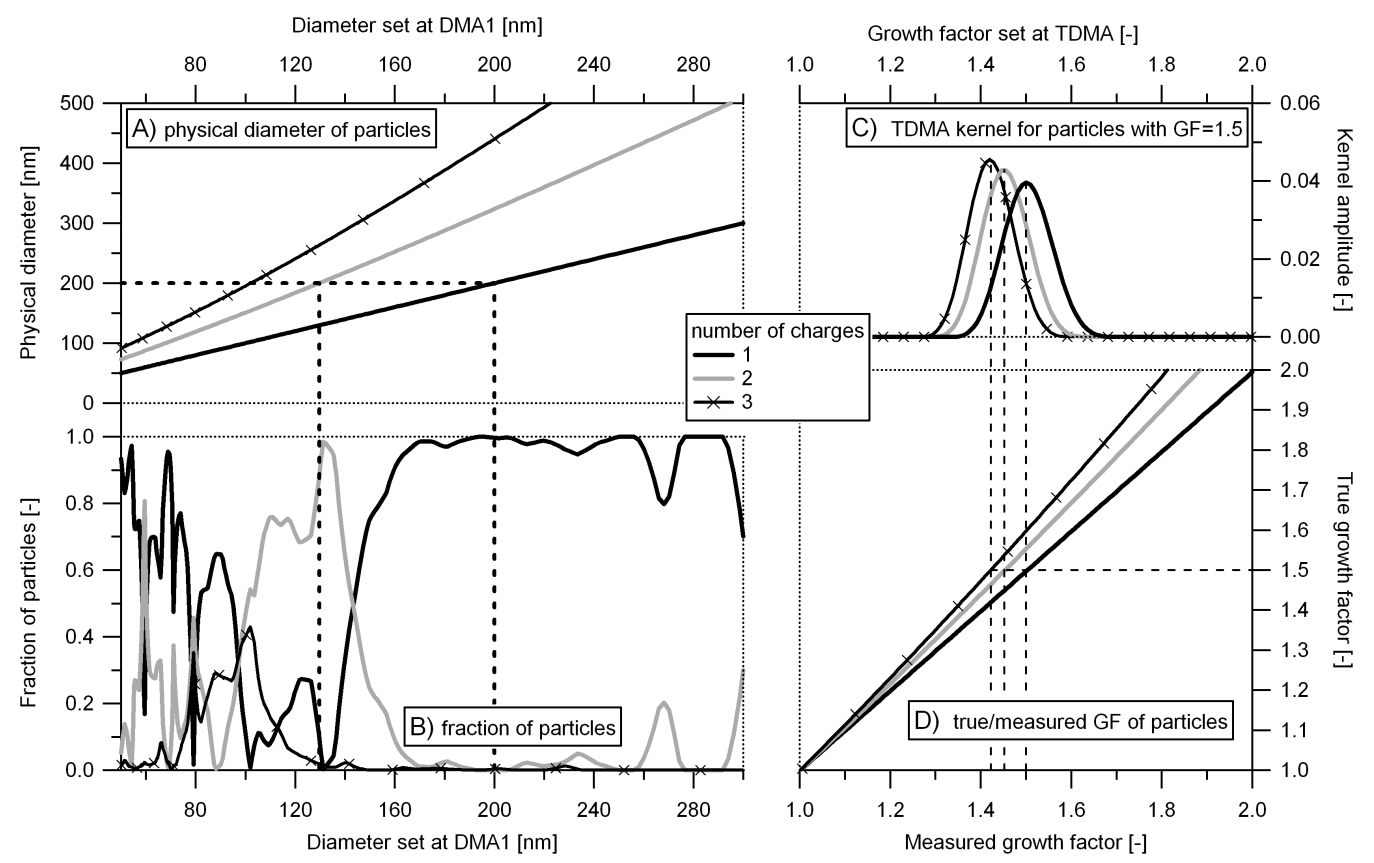

Fig. 9. Effects of particles carrying multiple charges; thick black, grey and crossed black lines represent singly, doubly and triply charges particles, respectively. A) The physical diameter of multiply charged particles is larger than the size set at DMA1. B) Number fraction of multiply charged particles versus electrical mobility diameter set at DMA1 as observed during a smogchamber experiment. Doubly charged particles dominate in the diameter range between $\sim 110-135 \mathrm{~nm}$, singly charged particles at diameters $>160 \mathrm{~nm}$ for the number size distribution observed in this particular experiment. C) TDMA kernel function for differently charged particles with equal electrical mobility diameter $\left(D_{0}=100 \mathrm{~nm}\right)$ and true $G F=1.5$. D) The mode of the MDF of mulitply charged particles peaks at a GF smaller than the true GF. 


\section{A Abbreviations and symbols}

CPC

Condensation particle counter.

DMA

Differential mobility analyser.

$\mathrm{FWHM}_{D}$

$\mathrm{FWHM}_{Z}$

GF

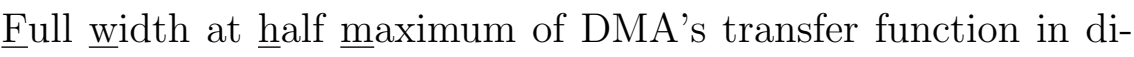
ameter space.

Full width at half maximum of DMA's transfer function in electrical mobility space.

GF-PDF

Growth factor.

Growth factor probability density function, $c\left(g, D_{0}\right)$, for particles with dry diameter $D=D_{0}$ to exhibit a $\mathrm{GF}=g$. The total probability of exhibiting a certain GF is unity: $\int_{0}^{\infty} c\left(g, D_{0}\right) d g=1$.
GF-CDF
MDF
Growth factor cumulative distribution function. The GF-CDF is the integral function of the GF-PDF (see Appendix C).
Measurement distribution function $m_{T D M A}^{\text {g,total }}\left(g_{\text {set }}, D_{0}\right)$. This is the particle concentration measured at the outlet of the TDMA as a function of GF, $g_{\text {set }}$, set at DMA2 for a defined dry size $\left(D_{0}\right)$ selected with DMA1.
R-MDF $\quad$ Reconstituted MDF. This is the calculated MDF obtained by sending the inverted GF-PDF back through the TDMA's forward function.
$\mathrm{RH}$
Relative humidity.
SMPS
Scanning mobility particle sizer.
TDMA
Tandem differential mobility analyser.
$c(g, D)$
See GF-PDF.
$\delta(x)$
Dirac's delta function.
$D$
Particle diameter.
$D_{0}$
Electrical mobility diameter set at DMA1 (dry diameter selected by DMA1).
$D_{i}$
Mobility diameter of a particle carrying $i$ charges with electrical mobility diameter $D_{0}: D_{i}=u_{i}\left(D_{0}\right)$.
$D_{\text {set }}$
$f_{z, i}(D)$
Electrical mobility diameter set at DMA2: $D_{\text {set }}=g_{\text {set }} D_{0}$
Function calculating the electrical mobility of a particle with dry diameter $D$ carrying $i$ charges. 


\begin{tabular}{|c|c|}
\hline$g$ & Diameter growth factor: $g=D / D_{0}$ \\
\hline$g_{\text {mean }}$ & Mean GF of the GF-PDF as defined in Eq. C.4. \\
\hline$g_{\text {set }}$ & GF set at DMA2: $g_{\text {set }}=D_{\text {set }} / D_{0}$ \\
\hline$\hat{g}$ & True GF of the particles (in terms of mobility diameter ratio). \\
\hline$K_{T D M A}^{g, i}\left(g_{\text {set }}, \hat{g}, D_{0}\right)$ & $\begin{array}{l}\text { TDMA kernel as a function of } g_{\text {set }} \text { and } D_{0} \text { for particles carrying } \\
i \text { charges and having a true } \mathrm{GF}=\hat{g} \text {. }\end{array}$ \\
\hline$k_{\text {inlet }}$ & $\begin{array}{l}\text { Factor to adjust the measurement uncertainty level due to vari- } \\
\text { ations of the particle concentration at the TDMA inlet. }\end{array}$ \\
\hline$k_{\text {kernel }}$ & $\begin{array}{l}\text { Factor to adjust the measurement uncertainty level due devia- } \\
\text { tions of the true kernel function from the kernel function used } \\
\text { in the inversion algorithm. }\end{array}$ \\
\hline$m_{T D M A}^{g, t o t a l}\left(g_{s e t}, D_{0}\right)$ & See MDF. \\
\hline$n$ & Number of counts in a single data point of the MDF. \\
\hline$n_{\max }$ & Maximum number of counts in any data point of the MDF. \\
\hline$n_{\text {total }}$ & Total number of counts recorded in the whole MDF. \\
\hline$\Delta n_{\text {poiss }}$ & $\begin{array}{l}\text { Uncertainty of the number of counts to account for Poisson } \\
\text { counting statistics. }\end{array}$ \\
\hline$\Delta n_{\text {inlet }}$ & $\begin{array}{l}\text { Uncertainty of the number of counts to account for variations of } \\
\text { the particle concentration at the TDMA inlet. }\end{array}$ \\
\hline$\Delta n_{\text {kernel }}$ & $\begin{array}{l}\text { Uncertainty of the number of counts introduced to account for } \\
\text { deviations of the true kernel function from the kernel function } \\
\text { used in the inversion algorithm. }\end{array}$ \\
\hline$\Delta n_{\text {overall }}$ & $\begin{array}{l}\text { Overall uncertainty of the number of counts in a data point of the } \\
\text { MDF obtained by combination of } \Delta n_{\text {poiss }}, \Delta n_{\text {inlet }} \text { and } \Delta n_{\text {kernel }} \text {. }\end{array}$ \\
\hline$N_{i n l e t}^{i}$ & $\begin{array}{l}\text { Normalisation factor for the TDMA kernel function for particles } \\
\text { carrying } i \text { charges: } N_{\text {inlet }}^{i}=p_{i}\left(D_{i}\right) \frac{d N_{\text {poly }}}{d \ln D}\left(D_{i}\right) .\end{array}$ \\
\hline$\frac{d N_{\text {poly }}}{d \ln D}$ & $\begin{array}{l}\text { Number size distribution of dried polydipserse aerosol at TDMA } \\
\text { inlet. }\end{array}$ \\
\hline$p_{i}(D)$ & $\begin{array}{l}\text { Number fraction of particles in the polydisperse aerosol with } \\
\text { diameter } D \text { carrying } i \text { charges. }\end{array}$ \\
\hline$Q_{s h}$ & Sheath air flow rate in the DMA. \\
\hline$Q_{e x}$ & Excess air flow rate in the DMA. \\
\hline$Q_{a e}$ & Polydisperse sample flow rate at DMA inlet. \\
\hline
\end{tabular}


$Q_{s a}$

$R\left(z, z_{\text {particle }}\right)$

$\sigma$

$T_{D M A}^{D, i}\left(D, D_{\text {particle }}\right)$ charges with diameter $D_{\text {particle }}$, which is defined as the probability of transfer of such particles when the DMA is set to select at diameter $D$.

$T_{D M A}^{g, i}\left(g, D_{\text {particle }}\right) \quad$ Diameter transfer function of a DMA for particles carrying $i$ charges with diameter $D_{\text {particle }}$, which is defined as the probability of transfer of such particles when the DMA is set to select at diameter $D=g D_{\text {particle }}$.

$u_{i}(D)$

$u_{i}^{-1}(D)$

$z$

Monodisperse sample flow rate at DMA outlet.

Electrical mobility transfer function of a DMA for particles with electrical mobility $z_{\text {particle }}$, which is defined as the probability of transfer of such particles when the DMA is set to select particles of electrical mobility $z$.

Standard deviation of the GF-PDF as defined in Eq. C.6.

Function to calculate the mobility diameter of a particle carrying $i$ charges and having equal electrical mobility as a singly charged particle with diameter $D$.

Function to calculate the mobility diameter of a singly charge particle having equal electrical mobility as a particle carrying $i$ charges with diameter $D$. This is the inverse function of $u_{i}(D)$.

Electrical mobility.

\section{B TDMA kernel and calibration}

\section{B.1 TDMA kernel and forward function}

The MDF, $m_{T D M A}^{g, t o t a l}\left(g_{\text {set }}, D_{0}\right)$, is the particle concentration at the TDMA sample outlet as a function of set GF, $g_{\text {set }}$, when DMA1 selects particles of single charge equivalent diameter $D_{0}$ and DMA2 selects at diameter $D_{\text {set }}=g_{\text {set }} D_{0}$. The MDF can be written as:

$$
m_{\text {TDMA }}^{\text {g,total }}\left(g_{\text {set }}, D_{0}\right)=
$$




$$
\sum_{i=1,2, \ldots} p_{i}\left(u_{i}\left(D_{0}\right)\right) \frac{d N_{\text {poly }}}{d \ln D}\left(u_{i}\left(D_{0}\right)\right) \int_{0}^{\infty} c\left(\hat{g}, u_{i}\left(D_{0}\right)\right) K_{T D M A}^{g, i}\left(g_{s e t}, \hat{g}, D_{0}\right) d \hat{g}
$$

where the summation is performed over the number of charges, $i$, on the particles. $c(g, D)$ is the GF-PDF of the particles with diameter $D$. The TDMA's kernel function $K_{T D M A}^{g, i}\left(g_{\text {set }}, \hat{g}, D_{0}\right)$ for particles carrying $i$ charges is defined as:

$K_{T D M A}^{g, i}\left(g_{\text {set }}, \hat{g}, D_{0}\right)=\int_{0}^{\infty} T_{D M A 1}^{g, i}\left(\frac{\hat{g}}{g^{\prime \prime}}, \frac{g^{\prime \prime}}{\hat{g}} D_{0}\right) \frac{1}{g^{\prime \prime}} T_{D M A 2}^{g, i}\left(\frac{g_{\text {set }}}{g^{\prime \prime}}, g^{\prime \prime} D_{0}\right) d g^{\prime \prime}$

where $T_{D M A}^{g, i}\left(g, D_{\text {particle }}\right)$ is the DMA's diameter transfer function as defined below. The kernel function is the normalised concentration of particles carrying $i$ charges with dry diameter $D_{i}=u_{i}\left(D_{0}\right)$, which is observed at the TDMA outlet, if DMA1 is set to diameter $D_{0}$, if DMA2 is set to diameter $D_{\text {set }}=g_{\text {set }} D_{0}$, if all particles of diameter $D_{i}$ exhibit a true GF of $\hat{g}$ (GF-PDF is a Dirac's delta function with peak at $\left.\hat{g}: c\left(g, D_{i}\right)=\delta(g-\hat{g})\right)$. The normalisation factor $N_{\text {inlet }}^{i}=p_{i}\left(D_{i}\right) \frac{d N_{\text {poly }}}{d \ln D}\left(D_{i}\right)$ is the number concentration of particles with charge $i$ in the relevant electrical mobility range at the inlet of the TDMA. With the above definitions, the complete TDMA forward function can be written as:

$$
m_{\text {TDMA }}^{\text {g,total }}\left(g_{\text {set }}, D_{0}\right)=\sum_{i=1,2, \ldots} N_{\text {inlet }}^{i}\left(D_{i}\right) \int_{0}^{\infty} c\left(\hat{g}, D_{i}\right) K_{\text {TDMA }}^{g, i}\left(g_{\text {set }}, \hat{g}, D_{0}\right) d \hat{g}
$$

The diameter transfer function can be written as:

$$
T_{D M A}^{g, i}\left(g, D_{\text {particle }}\right)=R\left(f_{z, 1}\left(g D_{\text {particle }}\right), f_{z, i}\left(D_{\text {particle }}\right)\right)
$$

where $R\left(z, z_{\text {particle }}\right)$ is the electrical mobility transfer function, which is defined as the transfer probability of a particle with electrical mobility $z_{\text {particle }}$, if the DMA is set to select at electrical mobility z. $f_{z, i}(D)$ is the function calculating the electrical mobility of a particle with dry diameter $D$ carrying $i$ charges. The idealised DMA electrical mobility transfer functions $R\left(z, z_{\text {particle }}\right)$ for equal sheath and excess flow rates is of symmetrical triangular shape, has a transfer probability of 1 at $z=z_{\text {particle }}$, and a constant full width at half maximum $\left(\mathrm{FWHM}_{z}\right)$ of $\Delta z / z_{\text {particle }}=\left(Q_{s a}+Q_{a e}\right) /\left(Q_{s h}+Q_{e x}\right)$, where $Q_{a e}, Q_{s a}, Q_{s h}$, and $Q_{e x}$ are the polydisperse sample, the monodisperse sample, the sheath, and the excess flow rates, respectively. The $\mathrm{FWHM}_{D}$ of the diameter transfer function $T_{D M A}^{g, i}$ depends on particle diameter due to the nonlinear relationship $f_{z, i}(D)$ between diameter and electrical mobility, but the shape remains virtually a symmetrical triangle with height one at $g_{\text {peak }}^{i}=u_{i}^{-1}\left(D_{\text {particle }}\right) / D_{\text {particle }}$. 
The function $u_{i}^{-1}(D)$ calculates the mobility diameter of singly charged particles having equal electrical mobility as particles carrying $i$ charges with diameter $D . u_{i}^{-1}(D)$ is the inverse function of $u_{i}(D)$. A consequence of the size-dependence of $\mathrm{FWHM}_{D}$ is a higher transfer probability for larger particles, causing a bias in the MDF towards larger GFs. Depending on the DMA's properties and operation settings, the actual transfer functions are somewhat broader and smoothed out due to various effects such as viscous flow, inhomogeneous electrical fields or particle diffusion (Collins et al., 2004; Hagwood et al., 1999; Stolzenburg, 1988). Eq. B.1 along with Eqs. B.2 and B.4 is the TDMA forward function, which fully describes the physical behaviour of the TDMA for given electrical mobility transfer functions $R\left(z, z_{\text {particle }}\right)$ of both DMAs. In many cases the number size distribution of the polydisperse aerosol is of such a shape that interferences from multiply charged particles are small in the TDMA measurements, such that Eq. B.1 can be reduced to the first term of the summation over the number of charges $i$ :

$$
m_{T D M A}^{g, 1}\left(g_{s e t}, D_{0}\right)=p_{1}\left(D_{0}\right) \frac{d N_{\text {poly }}}{d \ln D}\left(D_{0}\right) \int_{0}^{\infty} c\left(\hat{g}, D_{0}\right) K_{T D M A}^{g, 1}\left(g_{\text {set }}, \hat{g}, D_{0}\right) d \hat{g}
$$

Further conditions for the applicability of Eqs. B.1 and B.5 rely on the fact that DMA1's transfer function is sufficiently narrow: Firstly $c\left(g, D_{0}+\Delta D\right) \approx$ $c\left(g, D_{0}\right)$ and secondly $p_{1}\left(D_{0}+\Delta D\right) \frac{d N_{\text {poly }}}{d \ln D}\left(D_{0}+\Delta D\right) \approx p_{1}\left(D_{0}\right) \frac{d N_{\text {poly }}}{d \ln D}\left(D_{0}\right)$ for all $D_{0}+\Delta D$ within the support of $T_{D M A 1}^{D, 1}\left(D, D_{0}\right)$. The CPC used to record the measurement distribution has itself a finite response time to steep concentration changes, thus causing extra smearing of the recorded signal in case of fast scanning of the second DMA across $g_{\text {set }}$ (Weingartner et al., 2002). Therefore either sufficiently slow scanning or stepping of $g_{\text {set }}$ is to be applied at DMA2. Otherwise an extra inversion step removing the CPC's smearing effect from the recorded signal is required prior to further data analysis (e.g. Collins et al., 2002).

\section{B.2 TDMA kernel calibration}

\section{B.2.1 Offset calibration}

The TDMA kernel function $K_{T D M A}^{g, 1}\left(g_{\text {set }}, \hat{g}, D_{0}\right)$ of the TDMA must be calibrated because it is a key element of the data analysis. Fig. 2 shows an image plot of the theoretical kernel for a TDMA operated with an aerosol to sheath flow ratio of 1:10 in both DMAs and for singly charged particles with dry diameter $D_{0}=100 \mathrm{~nm}$. Unfortunately it is only possible to calibrate the TDMA response for particles exhibiting a true growth factor of $\hat{g}=1.0$ (dashed line in Fig. 2), which is only a one-dimensional cut, $K_{T D M A}^{g, 1}\left(g_{\text {set }}, \hat{g}=1, D_{0}\right)$, through 
the two-dimensional kernel function. Calibration of the TDMA for $\hat{g}=1.0$ (i.e. $c(g)=\delta(g-1))$, hereinafter referred to as offset calibration, can be performed by bypassing the treatment unit or by applying a 'null' treatment, which does not change the particle size. The latter can be achieved in a HTDMA by keeping the whole instrument at a $\mathrm{RH}$ clearly below the efflorescence point of the investigated particles. The mean GF of the inverted GF-PDF recorded during the offset calibration, $g_{o f f s e t}$, is typically up to a few percent different from 1.0 (see Fig. B.1A). The uncalibrated MDF is corrected for GF offsets by division of the GFs of all its data points by $g_{\text {offset }}$. This calibration assures that a GF of 1.0 is retrieved for those particles actually exhibiting a GF of 1.0. The TDMA response $K_{T D M A}^{g, 1}\left(g_{\text {set }}, \hat{g}=1.5, D_{0}\right)$ for particles exhibiting a GF of e.g. 1.5 (grey line in Fig. 2) cannot be calibrated because no treatment is available which makes all particles grow by an exactly known GF different from 1.0. HTDMA measurements of a pure salt do not fulfil this requirement mainly because the GF of the particles is strongly affected by $\mathrm{RH}$ uncertainties, which are larger than growth factor accuracy achieved with a properly operated TDMA. Proper TDMA operation means that the volumetric air flow rates through the DMAs are kept constant and that proper sizing of both DMAs over the relevant diameter range has been verified using e.g. certified monodisperse polystyrene latex spheres (PSL). Performing only offset calibrations at different $D_{0}$ without PSL verification of the sizing is not sufficient, because both DMA could suffer from equal nonlinear sizing errors, which were to result in $g_{\text {of } f \text { set }}=1.0$ at any size, thus giving no indication of the malfunction.

\section{B.2.2 Kernel width}

The MDF recorded for the offset calibration is also used to calibrate the TDMA kernel width, i.e. to determine the actual $\mathrm{FWHM}_{D}$ of the DMA transfer functions. The kernel calibration is done by variation of the $\mathrm{FWHM}_{D}$ of the DMA transfer functions until the corresponding $K_{T D M A}^{g, 1}\left(g_{\text {set }}, \hat{g}=1.0, D_{0}\right)$ matches the MDF of the offset calibration. Fig. B.1B shows an example, where the DMA's $\mathrm{FWHM}_{D}$ were found to be $\sim 1.066$ times wider than ideally expected, consistently across a range of different $D_{0}$. The broadening is assumed to occur in both DMAs equally because it is hardly possible to distinguish different broadening factors between the two DMAs.

\section{B.2.3 Concentration variations at TDMA inlet}

Offset calibrations allow to determine relative sizing offsets of the two DMAs (Appendix B.2.1) and to quantify the actual $\mathrm{FWHM}_{D}$ of the DMA transfer functions (Appendix B.2.2), because the GF-PDF is exactly known: $c(g)=$ $\delta(g-1)$. Differences between measured and calculated MDF remaining after 
the above calibrations can be attributed to either using an imprecise shape for the DMA transfer functions in the calculations or to counting uncertainties in the measurement as detailed in Sect. 2.3. The former effect is normally negligible. Therefore the latter effect can be used to estimate the magnitude of variations of the particle concentration at the TDMA's inlet from a series of offset calibrations. Our experience from several laboratory and field experiments indicates that $k_{\text {inlet }}$ is usually $\sim 5-10 \%$ behind aerosol generators and $\sim 5 \%$ for ambient measurements, though higher variability may be expected in environments with highly variable particle concentrations.

\section{Statistical definitions}

The GF-PDF, $c(g, D)$, is the GF probability density function for particles with dry diameter $D$, which describes the probability that these particles exhibit a certain GF, $g$, for a defined treatment. The inverted GF-PDFs are by definition normalised to a total probability of unity:

$$
\int_{0}^{\infty} c(g, D) d g=1
$$

The GF-CDF, $C(g, D)$, is the cumulative distribution function of GFs for particles with dry diameter $D$. It is the integral function of the GF-PDF:

$$
C(g, D)=\int_{0}^{g} c(\tilde{g}, D) d \tilde{g}
$$

The $\alpha$ percentile GF, $g_{\alpha}$, of the GF-PDF is defined such that:

$$
\alpha=C\left(g_{\alpha}, D\right)=\int_{0}^{g_{\alpha}} c(\tilde{g}, D) d \tilde{g}
$$

The number weighted $\left(1^{\text {st }}\right.$-moment) mean GF, $g_{\text {mean }}$, of a GF-PDF is defined as:

$$
g_{\text {mean }}=\int_{0}^{\infty} g c(g, D) d g
$$


The volume weighted ( $3^{r d}$-moment) mean GF, $g_{\text {mean }}^{\text {vol }}$, of a GF-PDF is defined as:

$$
g_{\text {mean }}^{\text {vol }}=\left(\int_{0}^{\infty} g^{3} c(g, D) d g\right)^{1 / 3}
$$

The GF standard deviation, $\sigma$, of a GF-PDF is defined as:

$$
\sigma=\left(\int_{0}^{\infty}\left(g-g_{\text {mean }}\right)^{2} c(g, D) d g\right)^{1 / 2}
$$

The growth spread factor, $S_{F}$, according to the definition by Stolzenburg and McMurry (1988) is:

$$
S_{F}=\frac{\sigma}{g_{\text {mean }}}
$$

The number fraction, $N^{a, b}$, of particles with $a<\mathrm{GF}<b$ is:

$$
N^{a, b}=\int_{a}^{b} c(g, D) d g
$$

The mean GF, $g_{\text {mean }}^{a, b}$, of the particles in a mode with $a<g<b$ is:

$$
g_{\text {mean }}^{a, b}=\frac{1}{N^{a, b}} \int_{a}^{b} g c(g, D) d g
$$

The GF standard deviation, $\sigma^{a, b}$, of the particles in a mode with $a<g<b$ is:

$$
\sigma^{a, b}=\left(\frac{1}{N^{a, b}} \int_{a}^{b}\left(g-g_{\text {mean }}^{a, b}\right)^{2} c(g, D) d g\right)^{1 / 2}
$$

The growth spread factor, $S_{F}^{a, b}$, of the particles in a mode with $a<\mathrm{GF}<b$ is:

$$
S_{F}^{a, b}=\frac{\sigma^{a, b}}{g_{\text {mean }}^{a, b}}
$$




\section{References}

Allan, J. D., Baumgardner, D., Raga, G. B., Mayol-Bracero, O. L., MoralesGarcía, F., García-García, F., Montero-Martínez, G., Borrmann, S., Schneider, J., Mertes, S., Walter, S., Gysel, M., Dusek, U., Frank, G. P., Krämer, M., 2008. Clouds and aerosols in Puerto Rico - a new evaluation. Atmos. Chem. Phys., 8 (5), 1293-1309.

Bilde, M., Pandis, S. N., 2001. Evaporation rates and vapor pressures of individual aerosol species formed in the atmospheric oxidation of $\alpha$ - and $\beta$ pinene. Environ. Sci. Technol. 35 (16), 3344-3349.

Cocker III, D. R., Whitlock, N. E., Flagan, R. C., Seinfeld, J. H., 2001. Hygroscopic properties of Pasadena, California aerosol. Aerosol Sci. Technol. 35 (2), 637-647.

Collins, D. R., Cocker, D. R., Flagan, R. C., Seinfeld, J. H., 2004. The scanning DMA transfer function. Aerosol Sci. Technol. 38 (8), 833-850.

Collins, D. R., Flagan, R. C., Seinfeld, J. H., 2002. Improved inversion of scanning DMA data. Aerosol Sci. Technol. 36 (1), 1-9.

Cubison, M., Coe, H., Gysel, M., 2005. Retrieval of hygroscopic tandem DMA measurements using an optimal estimation method. J. Aerosol Sci. 36 (7), 846-865.

Dassios, K. G., Pandis, S. N., 1999. The mass accommodation coefficient of ammonium nitrate aerosol. Atmos. Env. 33 (18), 2993-3003.

Dick, W. D., Saxena, P., McMurry, P. H., 2000. Estimation of water uptake by organic compounds in submicron aerosols measured during the Southeastern Aerosol and Visibility Study. J. Geophys. Res. 105 (D1), 1471-1479.

Duplissy, J., Gysel, M., Alfarra, M. R., Dommen, J., Metzger, A., Prevot, A. S. H., Weingartner, E., Laaksonen, A., Raatikainen, T., Good, N., Turner, F., McFiggans, G., Baltensperger, U., 2008. Cloud forming potential of secondary organic aerosol under near atmospheric conditions. Geophys. Res. Lett. 35, L03818, doi:10.1029/2007GL031075.

Gysel, M., Crosier, J., Topping, D., Whitehead, J., Bower, K., Cubison, M., Williams, P., Flynn, M., McFiggans, G., Coe, H., 2007. Closure study between chemical composition and hygroscopic growth of aerosol particles during TORCH2. Atmos. Chem. Phys. 7 (23), 6131-6144.

Gysel, M., Weingartner, E., Baltensperger, U., 2002. Hygroscopicity of aerosol particles at low temperatures. 2. Theoretical and experimental hygroscopic properties of laboratory generated aerosols. Environ. Sci. Technol. 36 (1), 63-68.

Hagwood, C., Sivathanu, Y., Mulholland, G., 1999. The DMA transfer function with Brownian motion a trajectory/Monte-Carlo approach. Aerosol Sci. Technol. 30 (1), 40-61.

Johnson, G. R., Ristovski, Z., Morawska, L., 2004. Method for measuring the hygroscopic behaviour of lower volatility fractions in an internally mixed aerosol. J. Aerosol Sci. 35 (4), 443-455.

Joutsensaari, J., Vaattovaara, P., Vesterinen, M., Hämeri, K., Laaksonen, A., 
2001. A novel tandem differential mobility analyzer with organic vapor treatment of aerosol particles. Atmos. Chem. Phys. 1 (1), 51-60.

Keith C. H., Arons, A. B., 1954. The growth of sea-salt particles by condensation of atmospheric water vapor. J. Meteorol. 11 (3), 173-184.

Knutson, E., Whitby, K. T., 1975. Aerosol classification by electric mobility: Apparatus, theory, and applications. J. Aerosol Sci. 6 (6), 443-451.

Liu, B. Y. H., Pui, D. Y. H., 1974. Submicron aerosol standard and primary, absolute calibration of the condensation nuclei counter. J. Colloid Interf. Sci. 47 (1), 155-171.

Liu, B. Y. H., Pui, D. Y. H., Whitby, K. T., Kittelson, D. B., Kousaka, Y., McKenzie, R. L., 1978. Aerosol mobility chromatograph - new detector for sulfuric-acid aerosols. Atmos. Env. 12 (1-3), 99-104.

McMurry, P. H., Takano, H., Anderson, G. R., 1983. Study of the ammonia (gas)-sulfuric acid (aerosol) reaction rate. Environ. Sci. Technol. 17 (6), $347-352$.

Meyer, N. K., Duplissy, J., Gysel, M., Metzger, A., Weingartner, E., Alfarra, M. R., Fletcher, C., Good, N., McFiggans, G., Jonsson, Å. M., Hallquist, M., Baltensperger, U., and Ristovski,Z. D., 2008. Analysis of the hygroscopic and volatile properties of ammonium sulphate seeded and un-seeded SOA particles. Atmos. Chem. Phys. Discuss., 8 (3), 8629-8659.

Paulsen, D., Weingartner, E., Alfarra, M. R., Baltensperger, U., 2006. Volatility measurements of photochemically and nebulizer-generated organic aerosol particles. J. Aerosol Sci. 37 (9), 1025-1051.

Philippin, S., Wiedensohler, A., Stratmann, F., 2004. Measurements of nonvolatile fractions of pollution aerosols with an eight-tube volatility tandem differential mobility analyzer (VTDMA-8). J. Aerosol Sci. 35 (2), 185-203.

Pitchford, M. L., McMurry, P. H., 1994. Relationship between measured water vapor growth and chemistry of atmospheric aerosol for Grand Canyon, Arizona, in winter 1990. Atmos. Env. 28, 827-839.

Rader, D. J., McMurry, P. H., 1986. Application of the tandem differential mobility analyzer to studies of droplet growth or evaporation. J. Aerosol Sci. 17 (5), 771-787.

Sjogren, S., Gysel, M., Weingartner, E., Alfarra, M. R., Duplissy, J., Cozic, J., Crosier, J., Coe, H., Baltensperger, U., 2008. Hygroscopicity of the submicrometer aerosol at the high-alpine site Jungfraujoch, 3580m a.s.l., Switzerland. Atmos. Chem. Phys., in press.

Stolzenburg, M., 1988. An ultrafine aerosol size distribution measuring system. Ph.D. thesis, University of Minnesota.

Stolzenburg, M., McMurry, P. H., February 1988. Tdmafit user's manual. Tech. Rep. PTL Publication No. 653, University of Minnesota, Department of Mechanical Engineering, Particle Technology Laboratory.

Stratmann, F., Kauffeldt, T., Hummes, D., Fissan, H., 1997. Differential electrical mobility analysis: A theoretical study. Aerosol Sci. Technol. 26 (4), 368-383.

Swietlicki, E., Hansson, H.-C., Hämeri, K., Svenningsson, B., Massling, A., Mc- 
Figgans, G., McMurry, P. H., Petäjä, T., Tunved, P., Gysel, M., Topping, D., Weingartner, E., Baltensperger, U., Rissler, J., Wiedensohler, A., Kulmala, M., 2008. Hygroscopic properties of submicrometer atmospheric aerosol particles measured with H-TDMA instruments in various environments - a review. Tellus, 60B (3), 432-469, doi:10.1111/j.1600-0889.2008.00350.x, 2008.

Voutilainen, A., Stratmann, F., Kaipio, J. P., 2000. A non-homogeneous regularization method for the estimation of narrow aerosol size distributions. J. Aerosol Sci. 31 (12), 1433-1445.

Weingartner, E., Gysel, M., Baltensperger, U., 2002. Hygroscopicity of aerosol particles at low temperatures. 1. New low-temperature H-TDMA instrument: Setup and first applications. Environ. Sci. Technol. 36 (1), 55-62. 


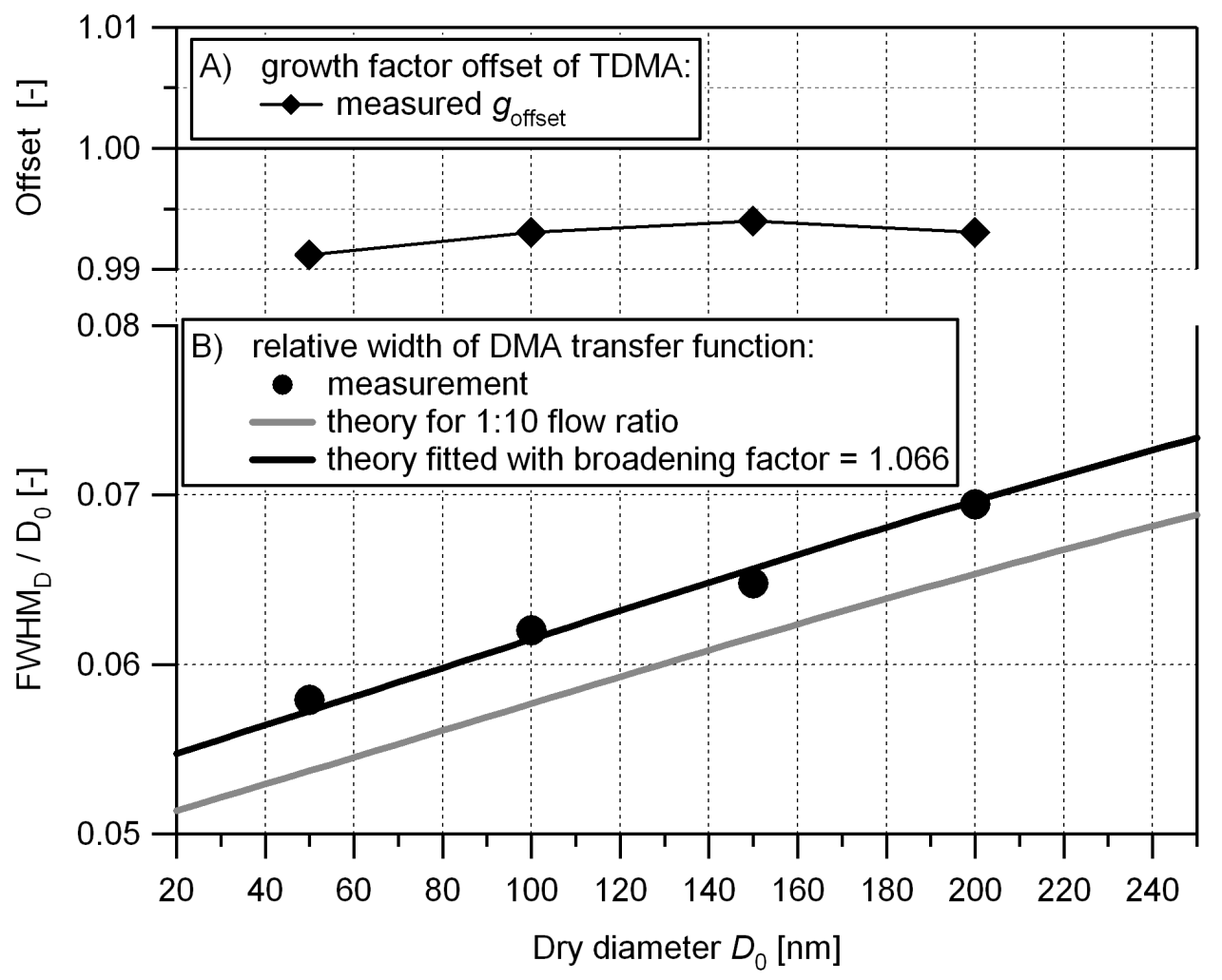

Fig. B.1. Offset calibration of TDMA. A) GF offset correction factor. B) Measured relative $\mathrm{FWHM}_{D} / D_{0}$ of DMAs compared with theory. 